\title{
A SURVEY OF THE WORK OF GEORGE LUSZTIG
}

\author{
R. W. CARTER
}

\section{Contents}

1. Introduction

2. The central theme

3. Early papers in representation theory

4. The Deligne-Lusztig paper of 1976

5. The Jordan decomposition of characters

6. The Harish-Chandra approach

7. Families of unipotent characters

8. The Kazhdan-Lusztig paper of 1979

9. The use of intersection cohomology

10. Perverse sheaves and character sheaves

11. Composition multiplicities of Verma modules

12. Representations of real Lie groups

13. Representations of $p$-adic reductive groups

14. The Springer correspondence and its affine version

15. Quantized enveloping algebras and their representations

16. The canonical basis

17. Total positivity in real reductive groups

18. Modular representations of simple algebraic groups

19. Modular representations of simple Lie algebras

20. Summary

\section{$\S 1$. Introduction}

It is an honour to be invited to contribute a survey article on the work of George Lusztig in celebration of his $60^{\text {th }}$ birthday.

George Lusztig came from the town of Timisoara in the mixed Romanian-Hungarian speaking part of Romania. He studied at the University of Bucharest and, after leaving Romania, worked with Michael Atiyah at the Institute for Advanced Study, Princeton for two years and also completed

Received October 18, 2005. 
a Ph. D. at Princeton University with William Browder as formal adviser. Subsequently he came to Warwick University, first as a postdoctoral research worker, then as a lecturer, and finally as a Professor. After seven years at Warwick he accepted a chair at M.I.T., where he has been working since.

Lusztig's exceptional mathematical ability became evident at an early stage of his career at Warwick. He gave a remarkable 30 lecture M. Sc. course on the modular representation theory of the general linear group in which, during the second half, he was working out the theory while giving the course. There were a few occasions when he was apologetic that the lecture lasted only 40 rather than 50 minutes because he had not made sufficient progress since the previous lecture!

His early experience as a mathematician was not without certain difficulties. There was a period during which, for financial reasons, he preferred to live in a tent outside the Mathematics Research Centre houses at Warwick University rather than in the houses themselves. He also experienced problems with the passport authorities in a number of countries so that, for a brief period, he had no entitlement to live in any country. However these difficulties did not prevent him from developing rapidly as a mathematician.

\section{$\S 2$. The central theme}

The central theme of Lusztig's mathematical work has been to approach the basic questions which arise in the representation theory of the structures which appear in Lie theory, such as reductive algebraic groups over the complex field, real field, $p$-adic fields, or finite fields; and also their Lie algebras, enveloping algebras and quantized enveloping algebras.

The methods he has used to achieve solutions to these basic questions include techniques from algebra and Lie theory, algebraic geometry and topology, including $\ell$-adic cohomology, intersection cohomology and equivariant $K$-theory.

The interrelation between different areas of mathematics is one of Lusztig's favourite themes, and his use of techniques from such diverse areas has led to quite spectacular success in determining the characters of the irreducible representations of the various Lie theoretical structures. We shall elaborate on this theme in the subsequent exposition. 


\section{§3. Early papers in representation theory}

After a number of early papers in topology published between 1965 and 1972, Lusztig's first substantial work on representation theory was his 1974 book on the discrete series of $G L_{n}$ over a finite field. The irreducible characters of the finite general linear groups $G L_{n}(q)$ had been obtained by J. A. Green in 1955. A key role in this theory is played by certain irreducible representations of degree $(q-1)\left(q^{2}-1\right) \cdots\left(q^{n-1}-1\right)$ called discrete series representations. Lusztig's work gives an explicit construction of a module giving a discrete series representation. Other discrete series representations are obtained by applying Galois automorphisms to this one. Lusztig's module is a free module over the Witt ring $W\left(\mathbb{F}_{q}\right)$ of the finite field $\mathbb{F}_{q}$, which is constructed by homological methods. Suppose $q$ is a power of the prime $p$. By using the properties of this module Lusztig was able to obtain an explicit Brauer lifting of the natural $G L_{n}(q)$-module $V$, i.e., a virtual representation of $G L_{n}(q)$ over $W\left(\mathbb{F}_{q}\right)$ whose reduction mod $p$ under the projection $W\left(\mathbb{F}_{q}\right) \rightarrow \mathbb{F}_{q}$ gives the modular character of $G L_{n}(q)$ on $V$.

A second work on representations of general linear groups also appeared in 1974, written in collaboration with R. W. Carter. The paper 'Modular representations of general linear and symmetric groups' by Carter and Lusztig studied the polynomial representations of $G L_{n}(k)$ over an infinite field $k$. When $k$ has characteristic 0 , there is a classical theory due to I. Schur in which the irreducible representations are obtained. They are in bijective correspondence with partitions with at most $n$ parts. When $k$ has prime characteristic $p$ the irreducible representations are still parametrised by such partitions but their dimensions are mainly unknown. Carter and Lusztig investigated modules called Weyl modules associated with such partitions. The irreducible modules are obtained as irreducible quotients of Weyl modules. It was shown that the structure of the Weyl modules is influenced by the affine Weyl group, which acts as a reflection group on the lattice of weights. For each pair of partitions related by a reflection in one of the reflecting affine hyperplanes and satisfying appropriate additional conditions, Carter and Lusztig proved the existence of a non-trivial homomorphism between the corresponding Weyl modules. Similar results were obtained about homomorphisms between Specht modules in the modular representation theory of symmetric groups.

The ideas introduced in this paper have proved quite influential in subsequent work on modular representations of reductive algebraic groups and symmetric groups. 


\section{$\S 4$. The Deligne-Lusztig paper of 1976}

There followed in 1976 a paper with P. Deligne which marked the fundamental breakthrough in understanding the irreducible characters over an algebraically closed field of characteristic 0 of reductive groups over finite fields. These groups include the finite Chevalley groups and their twisted analogues discovered by Steinberg, Tits, Suzuki and Ree. Such groups can be obtained as subgroups of connected reductive algebraic groups which are fixed by a Frobenius endomorphism or its variant.

Thus let $G$ be a connected reductive group over the algebraic closure $K$ of the field with $p$ elements. Any such group $G$ is isomorphic to a closed subgroup of $G L_{n}(K)$ for some $n$. Let $F_{q}$ be the endomorphism of $G L_{n}(K)$ given by $F_{q}\left(a_{i j}\right)=\left(a_{i j}^{q}\right)$ where $q$ is a power of $p$. A map $F: G \rightarrow G$ is called a Frobenius map if there exists an embedding of $G$ in $G L_{n}(K)$ such that $F$ is the restriction of $F_{q}$ for some $q$. Let $G^{F}$ be the subgroup of $F$-stable elements of $G$. The Deligne-Lusztig theory investigates the representations of the finite groups $G^{F}$.

A key role in this representation theory is played by the maximal tori of $G$ and $G^{F}$. A torus in $G$ is a closed subgroup isomorphic to a direct product of copies of the multiplicative group of $K$. Any two maximal tori of $G$ are conjugate. There exist $F$-stable maximal tori of $G$, and each $F$ stable maximal torus $T$ of $G$ gives a subgroup $T^{F}$ of $G^{F}$, called a maximal torus of $G^{F}$. The $F$-stable maximal torus $T$ of $G$ is called maximally split if it lies in an $F$-stable Borel subgroup (i.e. maximal soluble connected subgroup) of $G$. The Weyl group $W$ of $G$ is defined by $W=N(T) / T$ and is a finite Coxeter group. Assume that $T$ is maximally split. The $F$-action on $T$ gives rise to an $F$-action on $W$ and $w_{1}, w_{2} \in W$ are called $F$-conjugate if $w_{2}=w^{-1} w_{1} F(w)$ for some $w \in W$. Then there is a bijective correspondence between conjugacy classes of maximal tori $T^{F}$ in $G^{F}$ and $F$-conjugacy classes of $W$.

Let $\theta$ be a linear character of $T^{F}$ with values in an algebraically closed field of characteristic 0. Deligne and Lusztig showed how to obtain a generalized character of $G^{F}$ (i.e., a $\mathbb{Z}$-combination of irreducible characters) associated with $T$ and $\theta$. They defined this generalized character $R_{T, \theta}$ using the $\ell$-adic cohomology of certain algebraic varieties. If $\theta$ is a character of $T^{F}$ in general position then $\pm R_{T, \theta}$ is an irreducible character of $G^{F}$. This gives a number of families of irreducible characters of $G^{F}$, one family for each $G^{F}$-conjugacy class of maximal tori $T^{F}$, such that different families 
are disjoint. The degrees of these irreducible characters are

$$
\pm R_{T, \theta}(1)=\left|G^{F}: T^{F}\right|_{p^{\prime}}
$$

i.e., the part of the index of $T^{F}$ in $G^{F}$ which is prime to $p$.

If we consider characters $\theta$ of $T^{F}$ not necessarily in general position we obtain generalized characters $R_{T, \theta}$ of $G^{F}$ which can be expressed as $\mathbb{Z}$ combinations of irreducible characters. In fact each irreducible character of $G^{F}$ occurs as a component of some $R_{T, \theta}$. Two irreducible characters are said to be related if they are components of the same $R_{T, \theta}$. This relation generates an equivalence relation on the irreducible characters of $G^{F}$ which divides them into equivalence classes called Lusztig series.

We shall assume that the centre of $G$ is connected. Under this assumption each Lusztig series contains just one irreducible character whose degree is prime to $p$. Such irreducible characters are called the semisimple characters of $G^{F}$. The characters in the same Lusztig series as the unit character are called unipotent characters of $G^{F}$. The semisimple characters have elegant properties, for example there is a simple formula for their degrees, but the unipotent characters have turned out to be more difficult to understand.

\section{$\S 5$. The Jordan decomposition of characters}

Lusztig showed that a knowledge of the semisimple characters and unipotent characters of finite reductive groups gives a knowledge of all irreducible characters, by establishing a so-called Jordan decomposition of characters. This can be seen as analogous to the Jordan decomposition of elements. Given an element $g \in G^{F}$ there exists a unique semisimple element $s \in G^{F}$ and unipotent element $u \in G^{F}$ such that $g=s u=u s$. An element of $G^{F}$ is semisimple if and only if its order is prime to $p$ and unipotent if and only if its order is a power of $p$. Thus $g \in G^{F}$ determines a semisimple element $s \in G^{F}$ and an unipotent element in the centralizer of $s$.

In order to understand the Jordan decomposition of characters we need the concept of the dual group of $G$. For each connected reductive group $G$ there is a dual group $G^{*}$ in which roots and coroots are interchanged. $G^{*}$ is often called the Langlands dual of $G$. Given a Frobenius map $F: G \rightarrow G$ there is a corresponding Frobenius map $F^{*}: G^{*} \rightarrow G^{*}$ on the dual group. Thus we have a dual $\left(G^{*}\right)^{F^{*}}$ of our finite group $G^{F}$ and these two finite 
groups have the same order. Also there is a bijective correspondence between semisimple characters of $G^{F}$ and semisimple conjugacy classes of $\left(G^{*}\right)^{F^{*}}$. If $\chi_{s}$ is a semisimple character of $G^{F}$ corresponding to the semisimple conjugacy class containing $s^{*} \in\left(G^{*}\right)^{F^{*}}$ then we have

$$
\chi_{s}(1)=\left|\left(G^{*}\right)^{F^{*}}: C^{F^{*}}\right|_{p^{\prime}}
$$

where $C$ is the centralizer of $s^{*}$ in $G^{*}$. $C$ is also a connected reductive group.

Now suppose we are given an arbitrary irreducible character $\chi$ of $G^{F}$. Then there is a unique semisimple character $\chi_{s}$ in the same Lusztig series as $\chi$. Let $s^{*}$ be a corresponding semisimple element in $\left(G^{*}\right)^{F^{*}}$ with centralizer $C^{F^{*}}$. Then there is a bijective correspondence between characters in the Lusztig series containing $\chi_{s}$ and unipotent characters of $C^{F^{*}}$. In particular the character $\chi$ of $G^{F}$ corresponds to a unipotent character $\chi_{u}$ of $C^{F^{*}}$. The degrees of the characters $\chi, \chi_{s}, \chi_{u}$ are related by

$$
\chi(1)=\chi_{s}(1) \chi_{u}(1)
$$

This gives the Jordan decomposition of characters. The irreducible character $\chi$ of $G^{F}$ determines a semisimple character $\chi^{s}$ of $G^{F}$ and a unipotent character $\chi^{u}$ of the centralizer of a semisimple element of $\left(G^{*}\right)^{F^{*}}$ corresponding to $\chi^{s}$.

Since the semisimple characters of $G^{F}$ are rather well behaved, the Jordan decomposition of characters focussed attention on the unipotent characters. The main thrust of Lusztig's subsequent work on finite reductive groups was to gain an understanding of the unipotent characters.

\section{§6. The Harish-Chandra approach}

In order to obtain the unipotent characters of $G^{F}$ Lusztig made use of Harish-Chandra's ideas for obtaining irreducible characters of finite reductive groups by induction from parabolic subgroups. Any two Borel subgroups of the connected reductive group $G$ are conjugate, and a parabolic subgroup of $G$ is a subgroup which contains some Borel subgroup. Let $P$ be an $F$-stable parabolic subgroup of $G$. Then $P$ has a semidirect decomposition $P=U_{P} L$ where $U_{P}$ is the unipotent radical of $P$ and $L$ is a Levi subgroup of $P$. $L$ is also a connected reductive group, and it is possible to choose such a Levi subgroup which is also $F$-stable. Then $P^{F}$ has a semidirect product decomposition $P^{F}=U_{P}^{F} L^{F}$. 
Let $\phi$ be an irreducible character of $L^{F}$. Then $\phi$ can be lifted to a character $\phi_{P^{F}}$ of $P^{F}$ with $U_{P}^{F}$ in the kernel. This lifted character can be induced to $G^{F}$ to give the character $\operatorname{Ind}_{P^{F}}^{G^{F}} \phi$. The irreducible components of this induced character are irreducible characters of $G^{F}$. This is a method of obtaining irreducible characters of $G^{F}$ starting with an irreducible character of $L^{F}$, which will usually be smaller than $G^{F}$. An irreducible character $\chi$ of $G^{F}$ is called cuspidal if $\chi$ does not appear as a component of $\operatorname{Ind}_{P^{F}}^{G^{F}} \phi$ for any proper parabolic subgroup $P$ of $G$ and any irreducible character $\phi$ of $L^{F}$. Harish-Chandra's theorem asserts that given any irreducible character $\chi$ of $G^{F}$ there exists a parabolic subgroup $P^{F}$ of $G^{F}$ with Levi decomposition $P^{F}=U_{P}^{F} L^{F}$ and an irreducible cuspidal character $\phi$ of $L^{F}$ such that $\chi$ appears as a component of $\operatorname{Ind}_{P^{F}}^{G^{F}} \phi$. Moreover the pair $\left(L^{F}, \phi\right)$ is determined up to conjugacy.

The decomposition of the induced character $\operatorname{Ind}_{P F}^{G^{F}} \phi$ into irreducible components was determined by Howlett and Lehrer, who showed that these irreducible components are in natural bijective correspondence with the irreducible characters of a certain Coxeter group determined by $L$ and $\phi$.

In particular the unipotent characters of $G^{F}$ arise from cuspidal unipotent characters of $L^{F}$ by this process of lifting, inducing and decomposing. Lusztig obtained his results on unipotent characters by first determining the cuspidal unipotent characters and then applying the Harish-Chandra induction process. When $G$ is a classical group of type $A, B, C$ or $D$ then $G^{F}$ has at most one cuspidal unipotent character. In exceptional groups there can be more than one, and Lusztig displayed considerable virtuosity in determining them. A useful degree formula can be obtained for irreducible components $\chi$ of $\operatorname{Ind}_{P^{F}}^{G^{F}} \phi$ in terms of the degree of $\phi$ and the so-called generic degree of the irreducible character of the Coxeter group determined by $L$ and $\phi$. In this way Lusztig was able to obtain the degrees of all unipotent characters of $G^{F}$

\section{$\S 7$. Families of unipotent characters}

Having obtained the degrees of the unipotent characters of $G^{F}$ Lusztig found that these characters fall into families in a remarkable way. In order to describe this theory we shall assume for convenience that $G$ is simple and $G^{F}$ is of untwisted type, i.e., a Chevalley group. The results for twisted groups are broadly similar but more complicated to describe in detail.

We recall that an $F$-stable maximal torus $T$ of $G$ is maximally split if $T$ lies in an $F$-stable Borel subgroup $B$ of $G$. If $T$ is maximally split the 
Deligne-Lusztig generalized character $R_{T, 1}$ is the induced character $\operatorname{Ind}_{B^{F}}^{G^{F}} 1$. The irreducible components of this induced character are called unipotent characters in the principal series. The endomorphism algebra of the module with this induced character is isomorphic to the group algebra of the Weyl group $W$ of $G$. Thus there is a bijective correspondence between principal series unipotent characters of $G^{F}$ and irreducible characters of $W$.

The simplest case occurs when $G$ has type $A_{\ell}$. In this case every unipotent character of $G^{F}$ lies in the principal series. For any irreducible character $\phi$ of $W$ the corresponding unipotent character of $G^{F}$ is given by

$$
R_{\phi}=\frac{1}{|W|} \sum_{w \in W} \phi(w) R_{T_{w}, 1}
$$

where $T_{w}$ is an $F$-stable maximal torus of $G$ corresponding to the conjugacy class of $W$ containing $w$.

In general the situation is more complicated. Not every unipotent character of $G^{F}$ lies in the principal series. Also, given an irreducible character $\phi$ of $W$, the class function $R_{\phi}$ given above is not necessarily an irreducible character of $G^{F}$. However Lusztig showed that each unipotent character of $G^{F}$ is an irreducible component of $R_{\phi}$ for some irreducible character $\phi$ of $W$. By using this fact it is natural to introduce an equivalence relation on the unipotent characters. We say that two unipotent characters are related if they are components of a common class function $R_{\phi}$, and consider the equivalence generated by this relation. The equivalence classes obtained in this way are called families of unipotent characters of $G^{F}$.

Similarly the irreducible characters of $W$ may be divided into families. Two irreducible characters $\phi, \phi^{\prime}$ of $W$ are related if $R_{\phi}, R_{\phi^{\prime}}$ contain a common unipotent character of $G^{F}$ as component, and we consider the equivalence generated by this relation.

We now have a bijection between families of unipotent characters of $G^{F}$ and families of irreducible characters of $W$. It is possible to describe distinguished representatives both of the families of irreducible characters of $W$ and of the families of unipotent characters of $G^{F}$. For each irreducible character $\phi$ of $W$ we may define two polynomials $D_{\phi}(t)$ and $P_{\phi}(t) . D_{\phi}(t)$ is the polynomial such that the degree of the principal series unipotent character $\chi_{\phi}$ is $D_{\phi}(q) . P_{\phi}(t)$ is the polynomial such that the degree of $R_{\phi}$ is $P_{\phi}(q) . D_{\phi}(t)$ is called the generic degree polynomial and $P_{\phi}(t)$ the fake degree polynomial of $\phi$. $\phi$ was called by Lusztig a special character of $W$ if the highest power of $t$ dividing $D_{\phi}(t)$ and $P_{\phi}(t)$ is the same. It was 
shown by Lusztig that each family of irreducible characters of $W$ contains a unique special character $\phi$ and each family of unipotent characters of $G^{F}$ contains a unique character of form $\chi_{\phi}$ where $\phi$ is a special character of $W$.

The properties of the special characters can be used to determine the number of unipotent characters in each family, and also to give a formula for their degrees. Let $\phi$ be a special character of $W$. The generic degree $D_{\phi}(t)$ has form

$$
D_{\phi}(t)=c t^{a}+\text { terms of higher degree in } t
$$

where $c \neq 0$. There are only a few possible values for the constant $c$. If $G$ has type $A_{\ell} c$ is always equal to 1 . If $G$ has type $B_{\ell}, C_{\ell}$ or $D_{\ell} c$ has form $1 / 2^{e}$ for some $e \geq 0$. If $G$ has type $G_{2}, F_{4}, E_{6}, E_{7}, E_{8} c$ always takes one of the values

$$
1, \quad 1 / 2, \quad 1 / 6, \quad 1 / 24, \quad 1 / 120 \text {. }
$$

This led Lusztig to associate a certain finite group $\Gamma$ with the given special character $\phi . \Gamma$ lies in the class

$$
\left\{1, C_{2} \times \cdots \times C_{2}, S_{3}, S_{4}, S_{5}\right\}
$$

and is uniquely determined by the property

$$
c=1 /|\Gamma|
$$

This group $\Gamma$ gives the key to understanding the unipotent characters in the family containing $\chi_{\phi}$.

Let $x \in \Gamma$ and $C(x)$ be the centralizer of $x$. Let $\sigma$ be an irreducible character of $C(x)$ and $M$ be the set of pairs $(x, \sigma)$ modulo the action of $\Gamma$. We have

$$
|M|=1,2^{2 e}, 8,21,39
$$

for the above possible groups $\Gamma$. It was shown by Lusztig that the unipotent characters of $G^{F}$ in the family containing $\chi_{\phi}$ are parametrised by the elements of $M$. He also gave a formula for the degree of each unipotent character in the family in terms of a certain $|M| \times|M|$ matrix called the non-abelian Fourier transform matrix. (This non-abelian Fourier transform was rediscovered some ten years later by Physicists!). The class functions $R_{\phi}$ can be expressed in terms of the unipotent characters $\chi_{(x, \sigma)}^{\mathcal{F}}$ in the family $\mathcal{F}$ containing $\chi_{\phi}$ by means of this non-abelian Fourier transform matrix. 
For example the group $G^{F}=E_{8}(q)$ has 166 unipotent characters. These characters fall into 46 families; 23 of which contain 1 character, 18 contain 4 characters, 4 contain 8 characters, and 1 contains 39 characters.

The class functions $R_{\phi}$ are called almost characters. We consider $R_{\phi}=R\left(\chi_{\phi}\right)$ as the almost character corresponding to the principal series unipotent character $\chi_{\phi}$. Lusztig defined the almost character $R\left(\chi_{(x, \sigma)}^{\mathcal{F}}\right)$ for any unipotent character by an analogous formula. Then by using the Jordan decomposition of characters he defined an almost character $R(\chi)$ for any irreducible character $\chi$ of $G^{F}$. The almost characters form a basis of the space of class functions and, like the irreducible characters, satisfy the orthogonality relations. The almost characters have a geometrical significance which will appear later.

This remarkable theory of characters of finite reductive groups is undoubtedly one of the highlights of Lusztig's achievement. It is expounded in Lusztig's 1984 book 'Characters of Reductive Groups over a Finite Field'. Other useful expositions of this theory can be found in the books by R. W. Carter 'Finite groups of Lie type, Conjugacy classes and complex characters' and by F. Digne and J. Michel 'Representations of finite groups of Lie type'.

\section{§. The Kazhdan-Lusztig paper of $\mathbf{1 9 7 9}$}

The paper 'Representations of Coxeter groups and Hecke algebras' by D. Kazhdan and G. Lusztig in 1979 marked another fundamental breakthrough in understanding the representations of Lie theoretical structures, for in this paper the celebrated Kazhdan-Lusztig polynomials were defined and their significance explained.

The purpose of the paper is to construct certain representations of Hecke algebras. We again assume that $G^{F}$ is a finite Chevalley group with Borel subgroup $B^{F}$, and consider the induced representation $\operatorname{Ind}_{B^{F}} G^{F}(1)$ over $\mathbb{C}$. Let $H$ be the algebra of endomorphisms of the corresponding induced module. $H$ is called the Iwahori-Hecke algebra. The dimension of $H$ is the number of double cosets of $B^{F}$ in $G^{F}$, and this is equal to the order of the Weyl group $W . H$ has a basis $T_{w}$ for $w \in W$. Let $S$ be the set of fundamental reflections which generate $W$ as a Coxeter group. Then the multiplication of basis elements of $H$ is determined by the conditions

$$
\begin{aligned}
T_{s} T_{w} & =T_{s w} \quad \text { if } \ell(s w)=1+\ell(w) \\
T_{s}^{2} & =q T_{1}+(q-1) T_{s}
\end{aligned}
$$


for $s \in S, w \in W$, where $\ell$ is the length function on $W$ and $q$ is the number of elements in the finite field over which $G^{F}$ is taken.

This definition of an Iwahori-Hecke algebra can be generalized in a number of ways. In the first place $q$ can be replaced by an indeterminate $t$, to give the so-called generic Hecke algebra over $\mathbb{C}(t)$. Secondly it is convenient to introduce a square root of $t$ and to work over $\mathbb{C}\left(t^{1 / 2}\right)$. Thirdly one can restrict scalars to the ring

$$
A=\mathbb{Z}\left[t^{1 / 2}, t^{-1 / 2}\right]
$$

of Laurent polynomials in $t^{1 / 2}$ with integer coefficients. Finally one can replace the Weyl group $W$ by any Coxeter group. Thus we shall now consider $H$ to be a free $A$-module with basis $T_{w}$ and multiplication relations as above.

The idea of Kazhdan and Lusztig was to introduce a second basis $\left\{C_{w}\right\}$ of $H$ with striking properties. They first defined an involution $\theta$ on $H$. The base ring $A$ admits an involution $a \rightarrow \bar{a}$ interchanging $t^{1 / 2}$ and $t^{-1 / 2}$ and the involution $\theta$ on $H$ is defined by

$$
\theta\left(\sum a_{w} T_{w}\right)=\sum \bar{a}_{w}\left(T_{w^{-1}}\right)^{-1}
$$

Kazhdan and Lusztig obtained a basis of $\theta$-stable elements of $H$. They showed there exists a unique $C_{w} \in H$ such that

$$
\theta\left(C_{w}\right)=C_{w}
$$

and

$$
C_{w}=\sum_{y \leq w} \varepsilon(w) \varepsilon(y) t^{\frac{1}{2} \ell(w)-\ell(y)} P_{y, w}\left(t^{-1}\right) T_{y}
$$

where $\varepsilon(w)=(-1)^{\ell(w)}$ and $P_{y, w}(t) \in \mathbb{Z}[t]$ satisfies

$$
P_{w, w}(t)=1
$$

and

$$
\operatorname{deg} P_{y, w}(t) \leq \frac{1}{2}(\ell(w)-\ell(y)-1) \quad \text { if } y<w .
$$

Here $<$ is the Bruhat partial order on $W$ generated by $w<s w$ whenever $s$ is a (not necessarily simple) reflection in $W$ and $\ell(s w)=1+\ell(w)$.

$P_{y, w}(t)$ is called a Kazhdan-Lusztig polynomial and the $C_{w}$ form the Kazhdan-Lusztig basis of $H$. In particular we have $C_{1}=T_{1}$ and

$$
C_{s}=t^{-1 / 2} T_{s}-t^{1 / 2} T_{1} \quad \text { for } s \in S .
$$


We have the product rule

$$
C_{s} C_{w}= \begin{cases}C_{s w}+\sum_{\substack{z<w \\ s z<z}} \mu(z, w) C_{z} & \text { if } s w>w \\ \left(v+v^{-1}\right) C_{w} & \text { if } s w<w\end{cases}
$$

where $v=-t^{\frac{1}{2}}$ and $\mu(z, w)$ is the coefficient of $t^{\frac{1}{2}(\ell(w)-\ell(y)-1)}$ in $P_{y, w}(t)$. There is an analogous expression for $C_{w} C_{s}$.

We write $w \underset{L}{\rightarrow} w^{\prime}$ if $C_{s} C_{w}$ involves $C_{w^{\prime}}$ for some $s \in S$, and $w \underset{L}{\geq} w^{\prime}$ if there exist elements

$$
w=w_{1}, w_{2}, \ldots, w_{k}=w^{\prime}
$$

with $w_{i-1} \underset{L}{\rightarrow} w_{i}$ for each $i$. We write $w \underset{L}{\sim} w^{\prime}$ if $w \underset{L}{\geq} w^{\prime}$ and $w^{\prime} \underset{L}{\geq} w$. Then the relation $\underset{L}{\sim}$ is an equivalence relation on $W$ whose equivalence classes are called left cells. There is an analogous definition of right cells of $W$. We write $w \underset{L R}{\geq} w^{\prime}$ if there exist elements

$$
w=w_{1}, w_{2}, \ldots, w_{k}=w^{\prime}
$$

as above such that, for each $i$, either $w_{i-1} \underset{L}{\rightarrow} w_{i}$ or $w_{i-1} \underset{R}{\rightarrow} w_{i}$. We write $w \underset{L R}{\sim} w^{\prime}$ if $w \underset{L R}{\geq} w^{\prime}$ and $w^{\prime} \underset{L R}{\geq} w$. The relation $\underset{L R}{\sim}$ is an equivalence relation on $W$ whose equivalence classes are called two-sided cells.

Each left cell $X$ of $W$ gives rise to a left $H$-module $V_{X}$ defined as follows.

$$
V_{X}=\sum_{w \leq x} A C_{w} / \sum_{\substack{w<x \\ L}} A C_{w}
$$

where $w \underset{L}{<} x$ means $w \underset{L}{\leq} x$ and $w \underset{L}{\not} x$. This definition is independent of the choice of $x \in X . V_{X}$ has basis $C_{w}$ with $w \in X$ and gives rise to a representation of $H$ called a left cell representation.

By replacing the indeterminate $t$ by 1 we obtain left cell representations of the Coxeter group $W$. The left cell representations are not in general irreducible. However, when the Coxeter group $W$ is a Weyl group, each left cell representation contains a unique irreducible component which is a special representation of $W$, and two left cells lie in the same two-sided cell if and only if this special component is the same for both. Thus there is a natural bijection between special representations of $W$ and two-sided cell of $W$. This in turn gives a bijection between families of unipotent characters of a Chevalley group $G^{F}$ and two-sided cells of $W$. 


\section{$\S 9$. The use of intersection cohomology}

In a second paper of Kazhdan and Lusztig in 1980 it was shown that the Kazhdan-Lusztig polynomials $P_{y, w}(t)$ have a geometrical significance. This is related to the intersection cohomology of algebraic varieties. Intersection cohomology was originally introduced by Goresky and MacPherson in the context of topological spaces in order to provide an analogue of Poincaré duality for singular spaces. As part of their more general theory they defined middle intersection cohomology groups $I H^{i}(X)$ for any algebraic variety $X$ over $\mathbb{C}$.

An algebraic description of these middle intersection cohomology groups was subsequently given by Deligne. Let $X$ be an algebraic variety over $\mathbb{C}$ and $X_{\text {reg }}$ its set of non-singular points. We consider the category of bounded complexes of sheaves of $\mathbb{C}$-vector spaces on $X$. Let $D^{b} X$ be the derived category, whose objects are equivalence classes of such complexes under the relation of quasi-isomorphism. This relation preserves the cohomology sheaves of the complexes. Deligne defined an element $\mathbf{I C}^{\bullet}(X)$ of $D^{b} X$, the intersection cohomology complex, whose cohomology sheaves $\mathbf{I H}^{i}$ are constructible and which, on restriction to $X_{\text {reg }}$, gives the complex

$$
\cdots \longrightarrow 0 \longrightarrow 0 \longrightarrow \mathbb{C} \longrightarrow 0 \longrightarrow 0 \longrightarrow \cdots
$$

where the constant sheaf $\mathbb{C}$ appears in degree 0 . The hypercohomology groups of the intersection cohomology complex are the intersection cohomology groups of Goresky and MacPherson.

We now explain how the Kazhdan-Lusztig polynomials can be given in terms of intersection cohomology. Let $G$ be a simple algebraic group over $\mathbb{C}$ and $W$ be its Weyl group. Then $G$ has a Bruhat decomposition

$$
G=\bigcup_{w \in W} B w B
$$

into double cosets with respect to a Borel subgroup $B$. Let

$$
\mathcal{B}=G / B
$$

be the flag variety of $G$. We have

$$
\mathcal{B}=\bigcup_{w} \mathcal{B}_{w}
$$

where $\mathcal{B}_{w}=B w B / B$ is a Bruhat cell. The closure of $\mathcal{B}_{w}$ is given by

$$
\overline{\mathcal{B}}_{w}=\bigcup_{y \leq w} \mathcal{B}_{y} .
$$


$\overline{\mathcal{B}}_{y}$ is a Schubert variety.

We consider the intersection cohomology of this Schubert variety. Each point of $\overline{\mathcal{B}}_{w}$ lies in $\mathcal{B}_{y}$ for some $y \leq w$, and we consider the stalk of the intersection cohomology sheaf $\mathbf{I} \mathbf{H}^{i}$ at such a point. This stalk will be denoted by $\mathbf{I H}_{\mathcal{B}_{y}}^{i}\left(\overline{\mathcal{B}}_{w}\right)$, being independent of the choice of point in $\mathcal{B}_{y}$. It is known that

$$
\mathbf{I H}_{\mathcal{B}_{y}}^{2 i+1}\left(\overline{\mathcal{B}}_{w}\right)=0
$$

for all $i$. The geometric significance of the Kazhdan-Lusztig polynomials is given by the formula

$$
P_{y, w}(t)=\sum_{i \geq 0} \operatorname{dim} \mathbf{I H}_{\mathcal{B}_{y}}^{2 i}\left(\overline{\mathcal{B}}_{w}\right) t^{i} .
$$

In particular we see that when the Coxeter group $W$ is a Weyl group the coefficients of a Kazhdan-Lusztig polynomial $P_{y, w}(t)$ are non-negative integers.

The connection between representation theory and intersection cohomology revealed by the Kazhdan-Lusztig papers has proved to be of crucial importance. Lusztig has shown in subsequent papers that intersection cohomology is the key to solving a number of basic questions in different aspects of representation theory.

\section{$\S 10$. Perverse sheaves and character sheaves}

We next describe how ideas from intersection cohomology were used by Lusztig to give a geometrical approach to the study of irreducible representations of the finite reductive groups $G^{F}$. Lusztig defined a set of character sheaves on a connected reductive group $G$. This is a subclass of the class of objects in the category of perverse sheaves on $G$. The category of perverse sheaves on an algebraic variety $X$ was introduced and studied by Beilinson, Bernstein and Deligne in Astérisque 100. In order to describe the irreducible objects of this category we introduce some generalizations of the intersection cohomology complex $\mathbf{I C}^{\bullet}(X)$.

Deligne's description of the intersection cohomology complex on $X$ also makes sense for algebraic varieties over an algebraically closed field of characteristic $p$. In this case one takes $\ell$-adic sheaves on $X$ where $\ell$ is a prime

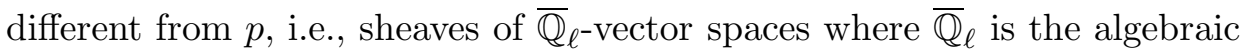
closure of the field of $\ell$-adic numbers.

Now let $X_{0}$ be an open dense non-singular subvariety of $X$ and $\mathcal{L}$ a local system on $X_{0}$. Thus $\mathcal{L}$ is a locally constant sheaf on $X_{0}$, and determines 
a representation of the fundamental group of $X_{0}$. Then one can define an object $\mathbf{I C}^{\bullet}(X, \mathcal{L})$ of $D^{b} X$ which, when restricted to $X_{0}$, gives the local system $\mathcal{L}$ in degree 0 and zero in other degrees. When $X_{0}=X_{\text {reg }}$ and $\mathcal{L}$ is the constant sheaf $\overline{\mathbb{Q}}_{\ell}$ we have

$$
\mathbf{I C}^{\bullet}(X, \mathcal{L})=\mathbf{I C}^{\bullet}(X) .
$$

More generally let $V$ be a locally closed non-singular irreducible subvariety of $X$ and $\mathcal{L}$ an irreducible local system on $V$. Then we have a complex $\mathbf{I C}^{\bullet}(\bar{V}, \mathcal{L})$ supported on the closure $\bar{V}$ of $V$ as above. This can be extended, by zero outside $\bar{V}$, to a complex on $X$ also denoted by $\operatorname{IC}^{\bullet}(\bar{V}, \mathcal{L})$. This is called the DGM-extension (i.e., Deligne, Goresky, MacPherson extension) of $\mathcal{L}$ to $X$. The elements of $D^{b} X$ obtained in this manner are, up to a degree shift, the irreducible objects in the category of perverse sheaves defined as follows.

There is a duality operation (Verdier duality)

$$
D: D^{b} X \longrightarrow D^{b} X .
$$

The complexes $\mathbf{K}$ in $D^{b} X$ satisfying the condition

$$
\operatorname{dim}\left(\operatorname{supp} H^{i}(\mathbf{K})\right) \leq-i
$$

for all $i \in \mathbb{Z}$ form a subcategory $D^{b} X^{\leq 0}$. The complexes $\mathbf{K}$ in $D^{b} X$ such that $D \mathbf{K} \in D^{b} X^{\leq 0}$ form a subcategory $D^{b} X^{\geq 0}$. Let $\mathcal{M} X$ be the full subcategory whose objects are the complexes $\mathbf{K}$ which lie in both $D^{b} X^{\leq 0}$ and $D^{b} X^{\geq 0}$. $\mathcal{M} X$ is called the category of perverse sheaves on $X$. It is an abelian category whose objects all have finite length. The duality operation maps $\mathcal{M X}$ to itself. The irreducible objects of $\mathcal{M} X$ are precisely the elements

$$
\mathbf{I C}^{\bullet}(\bar{V}, \mathcal{L})[\operatorname{dim} V]
$$

where $V$ is a locally closed non-singular irreducible subvariety of $X, \mathcal{L}$ is an irreducible local system on $V$, and $[\operatorname{dim} V]$ denotes degree shift by the dimension of $V$. These irreducible perverse sheaves are permuted by the operation of Verdier duality. In the case where a connected algebraic group $G$ acts on $X$, one can also consider $G$-equivariant perverse sheaves on $X$.

Lusztig showed how to introduce the concepts of Harish-Chandra theory into the category of $G$-equivariant perverse sheaves on a connected reductive group $G$ under the adjoint action. He defined the concept of a cuspidal perverse sheaf on $G$ and, if $P$ is a parabolic subgroup of $G$ with Levi 
decomposition $P=U_{P} L$, he showed how to define Harish-Chandra type functors of restriction and induction

$$
\begin{array}{r}
\operatorname{Res}: \mathcal{M} G \longrightarrow D^{b} L \\
\operatorname{Ind}_{P}^{G}: \mathcal{M} L \longrightarrow D^{b} G
\end{array}
$$

More precisely $\operatorname{Ind}_{P}^{G}$ is only defined on the $L$-equivariant objects of $\mathcal{M} L$.

It turns out that the cuspidal perverse sheaves are precisely the equivariant complexes $\mathbf{K}$ for which $\operatorname{Res} \mathbf{K}=0$ for all proper parabolic subgroups $P$ of $G$ and all Levi subgroups $L$ of $P$.

If $\mathbf{K}_{0}$ is a cuspidal perverse sheaf on $L$ then the induced object $\operatorname{Ind}_{P}^{G} \mathbf{K}_{0}$ is semisimple. A perverse sheaf on $G$ is called a character sheaf if it appears as an irreducible component of $\operatorname{Ind}_{P}^{G} \mathbf{K}_{0}$ for some parabolic subgroup $P$ of $G$ and some cuspidal perverse sheaf $\mathbf{K}_{0}$ of a Levi subgroup $L$ of $P$. Thus we have a Harish-Chandra theory on character sheaves analogous to that on finite reductive groups.

In his paper 'Intersection cohomology complexes on a reductive group' and his sequence of papers 'Character Sheaves I-V' Lusztig investigates in depth the character sheaves on any connected reductive group $G$. This theory is valid for groups $G$ over fields of arbitrary characteristic. However in the case when $G$ is a connected reductive group over the field $K=\overline{\mathbb{F}}_{p}$ the theory of character sheaves is shown to be relevant to the representation theory of the finite groups $G^{F}$ for Frobenius map $F: G \rightarrow G$. For a complex $\mathbf{K}$ on $G, F^{*} \mathbf{K}$ denotes the complex on $G$ obtained as the pull-back of $\mathbf{K}$ by $F$. In the case of a character sheaf $\mathbf{K}$ on $G$ for which there is an isomorphism $\phi$ between $\mathbf{K}$ and $F^{*} \mathbf{K}$ Lusztig defines a characteristic function

$$
\chi_{\mathbf{K}, \phi}: G^{F} \longrightarrow \overline{\mathbb{Q}}_{\ell} .
$$

Lusztig conjectured that, under mild assumptions on the characteristic $p$, these characteristic functions are scalar multiples of the almost characters of $G^{F}$ discussed in Section 7. He was able to prove this in certain cases in the papers 'On the character values of finite Chevalley groups at unipotent elements' Jour. Algebra 104 (1986), 146-194, where the conjecture was proved for the character sheaves whose supports contain unipotent elements in the case of adjoint groups of type $B_{n}$ and $E_{n}$; and 'Remarks on computing irreducible characters' Jour. Amer. Math. Soc. 5 (1992), 971-986, where the conjecture was proved for cuspidal character sheaves in large characteristic assuming the decomposition of Lusztig induction into irreducible characters 
(but without assuming the connectedness of the centre). This conjecture of Lusztig was subsequently proved by T. Shoji in his 1995 paper 'Character sheaves and almost characters of reductive groups' assuming that the centre of $G$ is connected. Thus the theory of character sheaves gives a geometrical construction of the almost characters of $G^{F}$. The irreducible characters of $G^{F}$ are obtained from the almost characters using the non-abelian Fourier transform matrix referred to in Section 7.

One of the most significant facts about character sheaves is that one can use them in computing irreducible character values of the finite reductive groups $G^{F}$. This is done by using an algorithm for computing the values of the characteristic functions of the $F$-stable character sheaves, based on the following ideas.

A deep result of Lusztig asserts the cleanness of character sheaves which are cuspidal. A consequence of this is that the values of the characteristic functions of the $F$-stable cuspidal character sheaves are very easy to compute. Using this fact, and the Jordan decomposition of elements of $G$, it is possible (though highly non-trivial) to reduce the determination of all values of the characteristic function of an $F$-stable character sheaf to that of values on unipotent elements. This in turn can be shown to involve the determination of certain functions known as generalized Green functions. A bridge connecting these generalized Green functions defined in terms of character sheaves and the analogous functions arising in the representation theory of the finite groups $G^{F}$ is constructed in Lusztig's paper 'Green functions and character sheaves'. Ann. Math. 131 (1990), 355-408.

Now there is an algorithm for computing the Green functions of $G^{F}$, based on an idea of Shoji in his 1983 paper 'On the Green polynomials of classical groups'. Lusztig found a modified (simpler) form of this algorithm and extended it to generalized Green functions in chapter 24 of his paper 'Character sheaves V'. A useful description of this algorithm can be found in Shoji's 1987 paper 'Green functions of reductive groups over a finite field'. In this way one can obtain an algorithm which will compute all values of the characteristic function of an $F$-stable character sheaf. This algorithm is now known to hold in almost complete generality, i.e., for all $p \neq 2$ and without restriction on the centre of $G$.

We now assume as before that $p$ is a good prime for $G$ and that $G$ has connected centre. Then by Shoji's theorem the characteristic functions of the $F$-stable character sheaves are scalar multiples of the almost characters of $G^{F}$. If the scalars were known, one would thus obtain the almost charac- 
ters of $G^{F}$, and hence the irreducible characters of $G^{F}$ using the non-abelian Fourier transform matrix. However this algorithm is not at present sufficient to obtain the irreducible characters of $G^{F}$, since the scalars involved are not known in general. These scalars are known in certain special cases, and in such cases all irreducible character values of $G^{F}$ can be obtained.

A good summary of the process of computing irreducible character values of $G^{F}$ can be found in the 1988 article 'Finite groups of Lie type' Section II, by M. Geck. In this paper Geck deals with the case in which $G$ is simple of adjoint type.

If the centre of $G$ is not connected the situation is more complicated. However progress has been made even in this case for certain classical groups by Bonnafé, Shoji and Waldspurger.

\section{§11. Composition multiplicities of Verma modules}

A remarkable application of the Kazhdan-Lusztig polynomials was conjectured by Kazhdan and Lusztig in 1979. This concerns the multiplicities of irreducible modules as composition factors of certain Verma modules.

Let $G$ be a simple algebraic group over $\mathbb{C}$ and $\mathfrak{g}$ be its Lie algebra. Then $\mathfrak{g}$ has a triangular decomposition

$$
\mathfrak{g}=\mathfrak{n}_{+} \oplus \mathfrak{h} \oplus \mathfrak{n}_{-}
$$

where $\mathfrak{h}$ is a Cartan subalgebra. Let

$$
\mathfrak{b}=\mathfrak{n}_{+} \oplus \mathfrak{h}
$$

Then $\mathfrak{b}$ is a Borel subalgebra of $\mathfrak{g}$. Let $\mathcal{U}(\mathfrak{g})$ be the universal enveloping algebra of $\mathfrak{g}$.

We take a weight of $\mathfrak{h}$, i.e., a linear map $\lambda: \mathfrak{h} \rightarrow \mathbb{C}$. This determines a 1-dimensional representation of $\mathfrak{b}$ with $\mathfrak{n}_{+}$in the kernel, and so a 1dimensional representation of the enveloping algebra $\mathcal{U}(\mathfrak{b})$. We may induce this representation to a $\mathcal{U}(\mathfrak{g})$-module $M_{\lambda}$ given by

$$
M_{\lambda}=\operatorname{Ind}_{\mathcal{U}(\mathfrak{G})}^{\mathcal{U}(\mathfrak{g})} \lambda .
$$

$M_{\lambda}$ is called the Verma module with highest weight $\lambda . M_{\lambda}$ has a unique maximal submodule $K_{\lambda}$, so the quotient

$$
L_{\lambda}=M_{\lambda} / K_{\lambda}
$$


is an irreducible $\mathcal{U}(g)$-module. $M_{\lambda}$ has a composition series of finite length, and each of its irreducible composition factors has form $L_{\mu}$ for some weight $\mu$.

Let $Z(\mathfrak{g})$ be the centre of $\mathcal{U}(\mathfrak{g})$. Then $Z(\mathfrak{g})$ acts on $M_{\lambda}$ by scalar multiplications, thus giving a 1-dimensional representation of $Z(\mathfrak{g})$ called the central character of $M_{\lambda}$. Two Verma modules $M_{\lambda}, M_{\mu}$ have the same central character if and only if

$$
\mu+\rho=w(\lambda+\rho)
$$

for some $w \in W$, the Weyl group, where $\rho$ is the sum of the fundamental weights of $\mathfrak{g}$. It follows that all composition factors of $M_{\lambda}$ have form $L_{w . \lambda}$ for some $w \in W$, where

$$
w \cdot \lambda=w(\lambda+\rho)-\rho .
$$

We now take a weight $\lambda$ which is integral, regular and antidominant, i.e.,

$$
(\lambda+\rho)\left(h_{i}\right) \in \mathbb{Z}, \quad(\lambda+\rho)\left(h_{i}\right)<0
$$

for each fundamental coroot $h_{i} \in \mathfrak{h}$. Then, as $w$ runs over the Weyl group, $w \cdot \lambda$ runs over a set of integral regular weights, one in each chamber with respect to the translated action of $W$.

We consider the multiplicities of the composition factors of $M_{w . \lambda}$. All such composition factors have form $L_{y . \lambda}$ for some $y \in W$ with $y \leq w$. Let $d_{y w}$ be the multiplicity of $L_{y \cdot \lambda}$ as a composition factor of $M_{w \cdot \lambda}$. The decomposition number $d_{y w}$ turns out to be independent of the choice of integral, regular, antidominant weight $\lambda$. Thus in a suitable Grothendieck group we have

$$
\left[M_{w . \lambda}\right]=\sum_{y \in W} d_{y w}\left[L_{y . \lambda}\right] .
$$

These equations may be inverted to give

$$
\left[L_{w . \lambda}\right]=\sum_{y \in W} a_{y w}\left[M_{y \cdot \lambda}\right] .
$$

for some $a_{y w} \in \mathbb{Z}$.

It was conjectured by Kazhdan and Lusztig that the integers $a_{y w}$ can be described in terms of Kazhdan-Lusztig polynomials, i.e., that

$$
a_{y w}=\varepsilon(y) \varepsilon(w) P_{y, w}(1) .
$$


The Kazhdan-Lusztig conjecture was proved in 1981 by Brylinski and Kashiwara, and independently by Beilinson and Bernstein. The proof involves a remarkable series of transitions of the problem, reformulating it in a number of different categories.

The Kazhdan-Lusztig polynomials can be interpreted in terms of the intersection cohomology of Schubert varieties, as described in Section 9. First of all we have an equivalence of categories

$$
\mathcal{M}_{1} \longleftrightarrow \mathcal{M}_{2}
$$

where $\mathcal{M}_{1}$ is a certain subcategory of holomorphic $D$-modules with regular singularities on the flag variety $\mathcal{B}$, and $\mathcal{M}_{2}$ is a certain subcategory of the category of $\mathfrak{g}$-modules. Moreover, by the general result on $D$-modules called the Riemann-Hilbert correspondence we have an equivalence of categories

$$
\mathcal{M}_{1} \longleftrightarrow \mathcal{M}_{3}
$$

where $\mathcal{M}_{3}$ is a certain subcategory of the category of perverse sheaves on $\mathcal{B}$. Combining these two equivalences we obtain an equivalence of categories

$$
\mathcal{M}_{2} \longleftrightarrow \mathcal{M}_{3}
$$

Now the $\mathfrak{g}$-modules $L_{w . \lambda}$ and $M_{y . \lambda}$ are objects of $\mathcal{M}_{2}$. Under the above equivalence $M_{y \cdot \lambda}$ corresponds to the constant sheaf $\mathbb{C}_{y}$ (shifted by $l(y)-$ $\operatorname{dim} \mathcal{B}$ ) on the Bruhat cell $\mathcal{B}_{y}$, whereas $L_{w . \lambda}$ corresponds to the intersection cohomology complex

$$
\mathbf{I C}^{\bullet}\left(\overline{\mathcal{B}}_{w}, \mathbb{C}_{w}\right)
$$

i.e., the DGM-extension of $\mathbb{C}_{w}$ to $\overline{\mathcal{B}}_{w}$. Thus the description of the KazhdanLusztig polynomials given in Section 9 gives rise to an equation in the category $\mathcal{M}_{3}$ which expresses $\mathbf{I C}^{\bullet}\left(\overline{\mathcal{B}}_{w}, \mathbb{C}_{w}\right)$ as a $\mathbb{Z}$-combination of $\mathbb{C}_{y}$ as $y$ runs over $W$. This is precisely the statement of the Kazhdan-Lusztig conjecture.

\section{§12. Representations of real Lie groups}

A somewhat similar idea was used by Lusztig and Vogan in 1981 to determine the characters of the irreducible modules for a real semisimple Lie group $G$ with trivial central character. Here we are considering irreducible admissible representations of $G$ on a Banach space. Such a representation has a character which is a distribution, called the Harish-Chandra character. The module giving each such irreducible representation occurs as a 
submodule of a module giving a so-called standard representation of $G$. The standard representations were constructed independently by Langlands and Zuckerman. One way of obtaining them is by a process of cohomological parabolic induction from characters of Cartan subgroups. The modules giving standard representations are not in general irreducible but have a unique minimal submodule. They have a composition series of finite length and each composition factor is isomorphic to the irreducible submodule of some other standard representation. The Harish-Chandra characters of the standard representations were known but, before the work of Lusztig and Vogan, the characters of the irreducible modules were not. We thus have a situation quite analogous to that of Verma modules.

Again in this situation it is possible to insert the equations expressing the characters of the standard representations in terms of those of the irreducible representations. Lusztig and Vogan showed that the characters of the irreducible modules could be expressed in terms of the characters of the standard representations by means of certain polynomials which are the analogues in the real Lie group situation of the Kazhdan-Lusztig polynomials.

\section{$\S 13$. Representations of $p$-adic reductive groups}

Lusztig then turned his attention to the representation theory of groups over $p$-adic fields or, more generally, over local fields.

Let $K$ be a non-Archimedean local field with finite residue field. Let $A$ be the ring of integers in $K$ and $\mathfrak{m}$ the unique maximal ideal of $A$. Let $k=A / \mathfrak{m}$ be the residue field. We assume that $k$ is a finite field with $q$ elements. There are two standard examples of this situation. Firstly we could take

$$
A=\mathbb{F}_{q}[[x]],
$$

the ring of formal power series over $\mathbb{F}_{q}$, and

$$
K=\mathbb{F}_{q}((x))
$$

its field of fractions. Secondly we could take $A=\mathbb{Z}_{p}$ to be the ring of $p$-adic integers and $K=\mathbb{Q}_{p}$ the field of $p$-adic numbers.

There are field extensions

$$
K \subset \tilde{K} \subset K_{\text {sep }} \subset \bar{K}
$$

where $\bar{K}$ is the algebraic closure of $K, K_{\text {sep }}$ its separable closure and $\tilde{K}$ the maximal unramified extension of $K$. Let $\tilde{A}$ be the ring of integers in $\tilde{K}$ and 
$\tilde{\mathfrak{m}}$ the unique maximal ideal of $\tilde{A}$. Let $\tilde{k}=\tilde{A} / \tilde{\mathfrak{m}}$. Then $\tilde{k}$ is isomorphic to the algebraic closure $\overline{\mathbb{F}}_{q}$ of $\mathbb{F}_{q}$. We have Galois groups

$$
\operatorname{Gal} \tilde{K} / K \cong \operatorname{Gal} \tilde{k} / k \cong \operatorname{Gal} \overline{\mathbb{F}}_{q} / \mathbb{F}_{q} \text {. }
$$

There is a Frobenius automorphism $F \in$ Aut $\tilde{K}$ uniquely determined by the properties

$$
F(\lambda)=\lambda \text { for all } \lambda \in K
$$

and $F(\lambda)=\lambda^{q}$ for $\lambda \in \tilde{A} / \tilde{\mathfrak{m}}=\tilde{k}$.

Now let $G$ be a simple algebraic group of adjoint type defined over $K$. Such a group is always quasi-split over $\tilde{K}$, but we shall assume that $G$ is split over $\tilde{K}$. We write

$$
G=G(\tilde{K})
$$

the group of rational points over $\tilde{K}$. The Frobenius map $F$ on $\tilde{K}$ induces a Frobenius map, also called $F$, on $G$. We have

$$
G^{F}=G(K),
$$

the group of $K$-rational points.

Lusztig considered admissible representations of $G^{F}$ over $\mathbb{C}$ (admissible representations are those which behave well on restriction to compact open subgroups). We have a natural homomorphism $\tilde{A} \rightarrow \tilde{k}$ which induces a homomorphism

$$
G(\tilde{A}) \longrightarrow G(\tilde{k})
$$

The inverse image of a Borel subgroup of $G(\tilde{k})$ is called an Iwahori subgroup of $G$. A subgroup of $G$ containing an Iwahori subgroup is called a parahoric subgroup.

Let $B$ be an Iwahori subgroup of $G$ and let $G^{\prime}=[G, G]$. Then $B \subset$ $G^{\prime}$ and there is a natural bijection between double cosets of $B$ in $G^{\prime}$ and elements of the affine Weyl group $W^{\prime}$ of $G$. $W^{\prime}$ is a Coxeter group whose Coxeter graph is the extended Dynkin diagram. We also have a bijection between double cosets of $B$ in $G$ and elements of the extended affine Weyl group $W . W$ is not in general a Coxeter group. Instead we have

$$
W=W^{\prime} \Omega, \quad W^{\prime} \cap \Omega=1
$$

where $\Omega$ is a finite abelian group which normalizes $W^{\prime} . \quad \Omega$ acts on the extended Dynkin diagram $I$ and is isomorphic to the group $S(I)$ of special automorphisms of $I$. 
Let $H$ be the Hecke algebra of the extended affine Weyl group $W$. The representations of this extended affine Hecke algebra $H$ turn out to be of key importance for the representation theory of groups over local fields. There is a bijective correspondence between irreducible admissible $G^{F}$-modules over $\mathbb{C}$ with a non-zero $B^{F}$-fixed vector, where $B$ is an $F$-stable Iwahori subgroup of $G$, and irreducible finite dimensional $H$-modules. These may be called the principal series unipotent representations of $G^{F}$. The DeligneLanglands conjecture asserted that these representations should correspond to pairs $(s, u)$ of elements in the dual group $G^{*}$ over $\mathbb{C}$, where $s$ is semisimple, $u$ is unipotent, and

$$
\text { sus }^{-1}=u^{q}
$$

such pairs being taken up to conjugacy in $G^{*}$. Lusztig formulated a more precise version of this conjecture, that the principal series unipotent representations of $G^{F}$ should be in bijective correspondence with triples $(s, u, \rho)$ with $s, u$ as above and where $\rho$ runs over a certain family of irreducible representations of the component group

$$
C(s, u) / C^{0}(s, u)
$$

of the centralizer of $s$ and $u$. This conjecture was proved by Kazhdan and Lusztig in 1987. The proof used methods of equivariant $K$-homology.

The use of equivariant $K$-theory and equivariant $K$-homology in representation theory is in fact one of Lusztig's major themes. The idea of using equivariant $K$-theory to attack the Deligne-Langlands conjecture was first formulated in Lusztig's 1985 paper on 'Equivariant $K$-theory and representations of Hecke algebras'. Equivariant $K$-theory may be used to determine the irreducible modules for affine Hecke algebras in which the parameters are all equal. However this method does not work when the parameters may be different, and this is precisely the case which one needs in the representation theory of $p$-adic groups.

In the case of affine Hecke algebras with unequal parameters Lusztig used a different method to determine the irreducible modules. In a series of papers 'Cuspidal local systems and graded Hecke algebras I, II, III' he introduced an infinitesimal version of the affine Hecke algebra called the graded affine Hecke algebra. This is the graded algebra associated with a certain filtration of the affine Hecke algebra. The relation between an affine Hecke algebra and its associated graded affine Hecke algebra is analogous to that between a reductive group and its Lie algebra. Lusztig was able 
to determine a parametrisation for the irreducible modules for the graded affine Hecke algebras, and to show that this parametrisation also applies to the irreducible modules of affine Hecke algebras with possibly different parameters. (In fact the graded affine Hecke algebra is the same as the 'degenerate affine Hecke algebra' which has appeared in recent papers of Cherednik, and which has significant applications in mathematical physics).

In a subsequent paper in 1995 Lusztig obtained much more far reaching results. He classified all irreducible admissible unipotent representations of $G^{F}$ in terms of triples $(s, u, \rho)$ coming from $G^{*}$. As before we have

$$
G=G(\tilde{K}), \quad G^{F}=G(K) .
$$

Let $P$ be an $F$-stable parahoric subgroup of $G$. $P$ has a prounipotent radical $U_{P}$ which is a projective limit of unipotent groups, and is also $F$ stable. Then $P^{F} / U_{P}^{F}$ is a finite group which is the subgroup of $F$-fixed points of a connected reductive group over $\mathbb{F}_{q}$. Thus the Deligne-Lusztig theory applies to

$$
P^{F} / U_{P}^{F}=\bar{P}^{F} \text {. }
$$

Let $E$ be a cuspidal unipotent $\bar{P}^{F}$-module, and let

$$
\operatorname{Irr}\left(G^{F} ; P, E\right)
$$

be the set of irreducible admissible $G^{F}$-modules such that the subspace of $U_{P}^{F}$-fixed vectors, regarded as a $\bar{P}^{F}$-module, contains a submodule isomorphic to $E$. A unipotent representation of $G^{F}$ is one which lies in $\operatorname{Irr}\left(G^{F} ; P, E\right)$ for some $F$-stable parahoric subgroup $P$ and some cuspidal unipotent $\bar{P}^{F}$-module $E$. If there is an element $g \in G^{F}$ which transforms $P$ to $P^{\prime}$ and $E$ to $E^{\prime}$ then

$$
\operatorname{Irr}\left(G^{F} ; P, E\right)=\operatorname{Irr}\left(G^{F} ; P^{\prime}, E^{\prime}\right)
$$

otherwise these two sets of representations have empty intersection. Thus the ideas of Harish-Chandra theory apply to the unipotent representations of $G^{F}$.

Now the Frobenius map $F$ on $G$ induces an automorphism of the extended Dynkin diagram $I$ which is a special graph automorphism, i.e., an element of $S(I)$. Also $S(I)$ is isomorphic to the group $\operatorname{Hom}\left(Z\left(G^{*}\right), \mathbb{C}^{*}\right)$ of central characters of the dual group $G^{*}$. Suppose the map $F \in S(I)$ corresponds to the central character $\chi$ of $G^{*}$. Then Lusztig showed that there 
is a bijective correspondence between irreducible unipotent representations of $G^{F}$ and triples $(s, u, \rho) \bmod G^{*}$, where $s \in G^{*}$ is semisimple, $u \in G^{*}$ is unipotent, sus ${ }^{-1}=u^{q}$, and $\rho$ is an irreducible representation of the component group $C(s, u) / C^{0}(s, u)$ on which $Z\left(G^{*}\right)$ acts as $\chi$. We shall call this Lusztig's duality theorem for reductive groups over local fields. The proof of this theorem is very remarkable, and to explain it we must first introduce some ideas related to the Springer correspondence.

\section{§14. The Springer correspondence and its affine version}

Let $G$ be a simple algebraic group over $\mathbb{C}$ with Weyl group $W$. T. A. Springer discovered a way of describing the irreducible representations of $W$. Let $C$ be a unipotent conjugacy class of $G$ and $u \in C$. Let $\mathcal{B}_{u}$ be the variety of Borel subgroups of $G$ containing $u$. Springer described an action of $W$ on the cohomology

$$
H^{2 d}\left(\mathcal{B}_{u}, \mathbb{C}\right)
$$

where $d=\operatorname{dim} \mathcal{B}_{u}$. The fact that $W$ acts on this cohomology module is at first sight surprising as $W$ does not act on $\mathcal{B}_{u}$ itself. Several different explanations of the $W$-action on the cohomology have since been discovered. The most natural and important of these is the one due to Lusztig in his paper 'Green polynomials and singularities of unipotent classes' using the methods of intersection cohomology. This action does not in general give an irreducible $W$-module. However the group

$$
A(u)=C(u) / C^{0}(u)
$$

also acts on $H^{2 d}\left(\mathcal{B}_{u}, \mathbb{C}\right)$ and the $A(u)$-action commutes with the $W$-action. Let $\rho$ be an irreducible $G$-equivariant local system on $C$. This corresponds to an irreducible representation $\rho$ of $A(u)$. The subspace

$$
H^{2 d}\left(\mathcal{B}_{u}, \mathbb{C}\right)_{\rho}
$$

on which $A(u)$ acts as $\rho$ is a $W$-submodule which decomposes into a direct sum of isomorphic irreducible $W$-modules. Every irreducible $W$-module arises in this way from such a pair $(C, \rho)$ and $(C, \rho)$ is determined up to conjugacy in $G$. However not every irreducible representation $\rho$ of $A(u)$ arises from $H^{2 d}\left(\mathcal{B}_{u}, \mathbb{C}\right)$. Thus we have a map

$$
\operatorname{Irr} W \longrightarrow\{(C, \rho)\}
$$


from irreducible characters of $W$ to pairs $(C, \rho)$ which is injective but not in general surjective. This map is called the Springer correspondence.

In his 1984 paper 'Intersection cohomology complexes on a reductive group' referred to in Section 10 Lusztig generalized the Springer correspondence. He showed that every pair $(C, \rho)$, where $C$ is a unipotent class in $G$ and $\rho$ a $G$-equivariant local system on $C$, determines a triple $\left(L, C^{\prime}, \rho^{\prime}\right)$ where $L$ is a Levi subgroup of some parabolic subgroup of $G, C^{\prime}$ is a unipotent class of $L$, and $\rho^{\prime}$ is an irreducible $L$-equivariant local system on $C^{\prime}$ which is cuspidal. Moreover the triple $\left(L, C^{\prime}, \rho^{\prime}\right)$ is determined up to $G$-conjugacy. The set of pairs $(C, \rho)$ which correspond to a fixed triple $\left(L, C^{\prime}, \rho^{\prime}\right)$ are in natural bijective correspondence with irreducible representations of the relative Weyl group

$$
W(G ; L)=N_{G}(L) / N_{G}(L)^{0} .
$$

$W(G, L)$ is not in general a Coxeter group, but it is a Coxeter group in the case of a Levi subgroup $L$ which possesses a pair $\left(C^{\prime}, \rho^{\prime}\right)$ with $\rho^{\prime}$ cuspidal. This gives rise to a bijection between the set of pairs $\{(C, \rho)\}$ and the set

$$
\bigcup \operatorname{Irr} W(G ; L)
$$

with one term for each cuspidal triple $\left(L, C^{\prime}, \rho^{\prime}\right)$. In the special case $L=T$, a maximal torus, we have $W(G ; T)=W$ and obtain the original Springer correspondence. The above bijection is called the generalized Springer correspondence.

In order to prove his duality theorem for groups over local fields Lusztig obtained an affine version of the generalized Springer correspondence. Here the pairs $(C, \rho)$ are replaced by triples $(s, u, \rho)$ where

$$
\begin{aligned}
& s \in G \text { is semisimple } \\
& u \in G \text { is unipotent } \\
& s u=u s \\
& \rho \text { is an irreducible representation of } C(s, u) / C(s, u)^{0} .
\end{aligned}
$$

The Coxeter group $W(G ; L)$ is replaced by an extended affine Weyl group

$$
W_{a}(G ; L) \text {. }
$$

There is a bijective correspondence

$$
\{(s, u, \rho)\} \bmod G \longleftrightarrow \bigcup_{\left(L, C^{\prime}, \rho^{\prime}\right) \bmod G} \operatorname{Irr} W_{a}(G ; L)
$$


and this is the affine version of the generalized Springer correspondence.

Lusztig also proved a $q$-version of this result in which each extended affine Weyl group is replaced by the corresponding extended affine Hecke algebra with parameter $q \in \mathbb{C}$, which is not a root of unity. The pairs $(s, u)$ with $s u=u s$ are replaced by pairs $(s, y)$ where

$$
\begin{aligned}
& s \in G \text { is semisimple } \\
& y \in \operatorname{Lie} G \text { is nilpotent } \\
& \operatorname{Ad}(s) y=q y .
\end{aligned}
$$

The $q$-version of the affine generalized Springer correspondence asserts that there is a bijection between triples $(s, y, \rho) \bmod G$, where $\rho$ is an irreducible representation of

$$
C(s, y) / C^{0}(s, y)
$$

and the set

$$
\bigcup_{\left(L, C^{\prime}, \rho^{\prime}\right)} \operatorname{Irr} H(G ; L)
$$

where $H(G ; L)$ is an extended affine Hecke algebra with parameter $q$. There is one component for each cuspidal triple $\left(L, C^{\prime}, \rho^{\prime}\right) \bmod G$. The quadratic relations for $H(G ; L)$ have form

$$
T_{s_{i}}^{2}=q^{f(i)} T_{1}+\left(q^{f(i)}-1\right) T_{s_{i}}
$$

where $f$ is a certain function on the Coxeter generators $s_{i}$ taking constant values on conjugate generators. This affine $q$-version of the generalized Springer correspondence was proved by Lusztig in his 1995 paper on the 'Classification of unipotent representations of simple $p$-adic groups', although some of the ideas involved in the proof can be traced back to earlier papers of Lusztig.

We now return to our simple algebraic group $G$ of adjoint type defined over the local field $K$. We have

$$
G=G(\tilde{K}), \quad G^{F}=G(K) .
$$

The unipotent representations of $G^{F}$ are those which lie in

$$
\operatorname{Irr}\left(G^{F} ; P, E\right)
$$


where $P$ is an $F$-stable parahoric subgroup of $G$ and $E$ is a cuspidal unipotent $\bar{P}^{F}$-module. One can define an induced $G^{F}$-module $\operatorname{Ind}_{P^{F}}^{G^{F}} E$. It was shown by Moy and Prasad that there is a natural bijection between unipotent representations in $\operatorname{Irr}\left(G^{F} ; P, E\right)$ and finite dimensional irreducible representations of the endomorphism algebra

$$
H=\operatorname{End}_{G^{F}}\left(\operatorname{Ind}_{P F}^{G^{F}} E\right) .
$$

This endomorphism algebra $H$ can be shown to be an extended affine Hecke algebra whose underlying Coxeter group is a relative affine Weyl group of $G$ with respect to $P$. Thus each unipotent representation of $G^{F}$ corresponds to an irreducible finite dimensional representation of one of the extended affine Hecke algebras $H=H(P, E)$.

On the other hand we may consider the set of triples $(s, y, \rho)$ in the dual group $G^{*}$ where

$$
\begin{aligned}
& s \in G^{*} \text { is semisimple } \\
& y \in \operatorname{Lie} G^{*} \text { is nilpotent } \\
& \operatorname{Ad}(s) y=q y .
\end{aligned}
$$

$\rho$ is an irreducible representation of $C(s, y) / C^{0}(s, y)$ on which $Z\left(G^{*}\right)$ acts by the central character $\chi$ introduced in Section 13 .

By the $q$-version of the affine generalized Springer correspondence, such triples taken $\bmod G^{*}$ are in bijective correspondence with

$$
\bigcup_{\left(L, C^{\prime}, \rho^{\prime}\right)} \operatorname{Irr} H\left(G^{*} ; L\right)
$$

where each $H\left(G^{*}, L\right)$ is an extended affine Hecke algebra and we have one component for each cuspidal triple $\left(L, C^{\prime}, \rho^{\prime}\right)$ in $G^{*}$.

Thus the objects on both sides of Lusztig's duality theorem for groups over local fields are parametrised by irreducible representations of extended affine Hecke algebras. One therefore tries to compare the extended affine Hecke algebras which appear on both sides. In order to describe such an algebra one must specify the underlying affine Weyl group, the function $f$ on the Coxeter generators giving the quadratic relations, and the finite abelian group $\Omega$ acting on the affine Weyl group and preserving the function $f$. When this comparison is made between the extended affine Hecke algebras appearing on both sides of Lusztig's duality theorem it transpires that they match exactly! This is proved in a case by case manner as there is at 
present no theoretical explanation of this matching. The way in which the matching works is truly remarkable, and results in the proof of Lusztig's duality theorem. This proof works for each inner form of a split group over a non-archimedean local field. We illustrate this matching in the group $E_{8}\left(\mathbb{F}_{q}((x))\right)$. A further substantial paper by Lusztig in 2002 extends this duality theorem to groups which are not necessarily inner forms of split groups.

An example: $G^{F}=E_{8}\left(\mathbb{F}_{q}((x))\right), G^{*}=E_{8}(\mathbb{C})$.

\section{Unipotent representations of $G^{F}$}

$$
P \quad \text { Diagram No. of cuspidal E's Hecke algebra }
$$
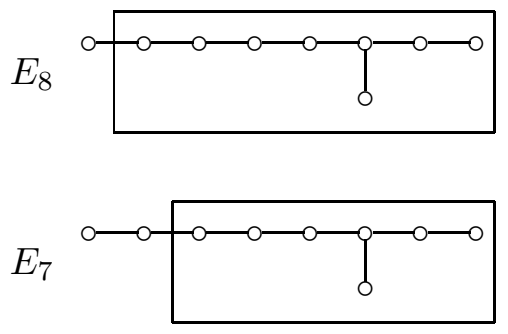

$E_{6}$



2

$\stackrel{\circ \longrightarrow q}{\rightleftharpoons} \underset{q}{\rightleftharpoons} q^{9}$

1

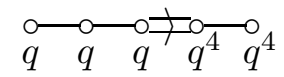

$D_{4}$

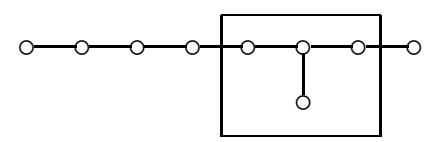

B

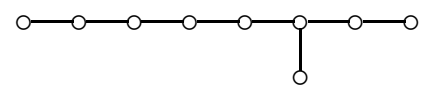

1

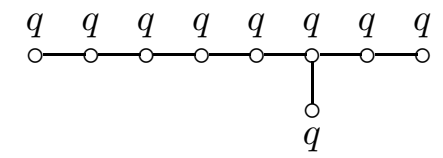

$\tilde{G}_{2}$

$\tilde{F}_{4}$

$\tilde{E}_{8}$

We have omitted the finite abelian group $\Omega$ for simplicity. 
Triples $(s, u, \rho)$ from $E_{8}(\mathbb{C})$

$L$ Diagram No. of cuspidal $\rho^{\prime}$ Hecke algebra $E_{8}$

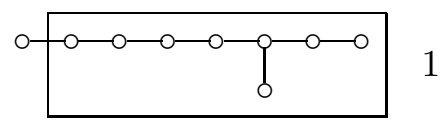
$\operatorname{dim} 1$ $E_{7} A_{1}$

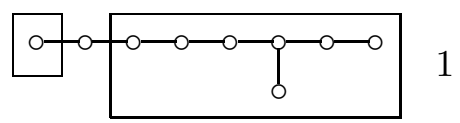
$\operatorname{dim} 1$ $E_{6} A_{2}$

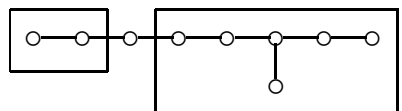
$\operatorname{dim} 1$ $D_{5} A_{3}$

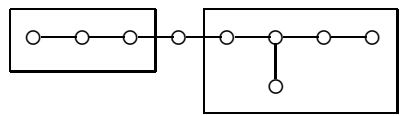
$\operatorname{dim} 1$ $A_{4} A_{4}$


$A_{5} A_{2} A_{1}$

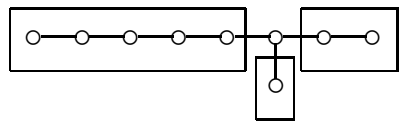<smiles>c1ccncc1</smiles>

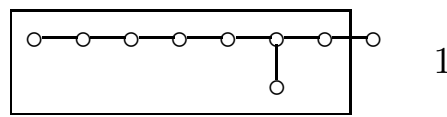
$\operatorname{dim} 1$

$A_{3} A_{3} A_{1}$

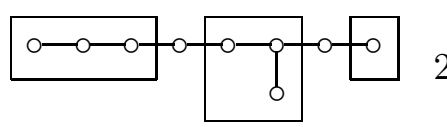

${ }^{q} q^{15}$ $\tilde{A}_{1}$ $A_{2} A_{2} A_{2}$

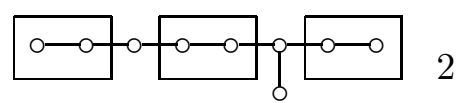

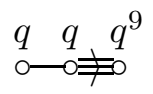
$\tilde{G}_{2}$

$A_{1} A_{1} A_{1} A_{1}$

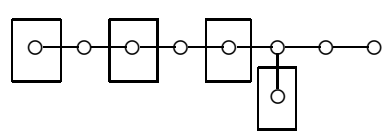

1<smiles></smiles> 


\section{$\S 15$. Quantized enveloping algebras and their representations}

In 1988 Lusztig turned his attention to the theory of quantized enveloping algebras. These are also known as quantum groups, although the term quantum group is also frequently used for a more general type of mathematical structure. Quantized enveloping algebras were defined independently by V. Drinfeld and M. Jimbo in 1985, although the special case of quantum groups of type $s l_{2}$ had been studied earlier in 1981 by Kulisch and Reshetikhin, and by Sklyanin. Quantized enveloping algebras are quantum deformations of enveloping algebras of simple Lie algebras over $\mathbb{C}$. Lusztig's first contribution was to observe that the irreducible integrable highest weight modules for the enveloping algebra admit quantum deformations which give irreducible modules for the quantized enveloping algebra. Subsequently, in 1989, he considered quantized enveloping algebras in which the indeterminate parameter $v$ is replaced by a root of unity. He first defined an integral form over $\mathbb{C}\left[v, v^{-1}\right]$ of the original algebra $U$ over $\mathbb{C}(v)$. This is analogous to Kostant's integral form $\mathcal{U}_{\mathbb{Z}}$ of the enveloping algebra $\mathcal{U}$. Then he considered the algebra $U_{\zeta}$ obtained from the integral form by specializing the parameter $v$ to a primitive $\ell^{\text {th }}$ root of unity $\zeta$. For each $n$-tuple $\lambda$ of non-negative integers Lusztig obtained a $U$-module $M(\lambda)$ analogous to a Verma module. This has a unique maximal submodule giving rise to an irreducible $U$-module quotient $V(\lambda)$, analogous to a Weyl module. By using the integral form he constructed an analogous module $V_{\zeta}(\lambda)$ for $U_{\zeta} . V_{\zeta}(\lambda)$ is a finite dimensional module whose character is known. It has a unique maximal submodule, thus giving an irreducible $U_{\zeta}$-module quotient $L_{\zeta}(\lambda)$.

Lusztig gave a conjecture for the character of the irreducible module $L_{\zeta}(\lambda)$ as a linear combination of characters of Weyl modules $V_{\zeta}(\mu)$. This is somewhat similar to the Kazhdan-Lusztig conjecture, discussed in Section 11. However this time the coefficients involve Kazhdan-Lusztig polynomials evaluated at 1 , not of the Weyl group but of the affine Weyl group.

Lusztig's conjecture for characters of irreducible modules for the quantum groups $U_{\zeta}$ suggested that there is a connection between the representation theory of quantum groups at a root of unity and the representation theory of affine Kac-Moody algebras. This connection was made precise in a paper of Kazhdan and Lusztig in 1991 in which an equivalence of tensor categories was defined between certain categories of $U_{\zeta}$-modules and modules with a particular negative central change for the corresponding affine Kac-Moody algebra. The proof that the functor defined by Kazhdan and 
Lusztig is indeed an equivalence of tensor categories is very elaborate, and was given in a series of subsequent papers by Kazhdan and Lusztig.

Once this equivalence of tensor categories has been established, Lusztig's conjecture on the irreducible characters of $U_{\zeta}$-modules becomes equivalent to an affine version of the original Kazhdan-Lusztig conjecture for Verma modules. This affine version was subsequently proved by M. Kashiwara and T. Tanisaki. Thus Lusztig's conjectured formula for the characters of the irreducible $U_{\zeta}$-modules $L_{\zeta}(\lambda)$ has turned out to be true.

\section{§16. The canonical basis}

In 1990 Lusztig made another discovery of fundamental importance by proving the existence of a remarkable basis of a quantized enveloping algebra called the canonical basis. (This basis was also independently proved to exist by Kashiwara using a different method, and Grojnowski and Luzstig proved that the two bases are the same).

Let

$$
U=U^{-} \otimes U^{0} \otimes U^{+}
$$

be a quantized enveloping algebra over the field of rational functions $\mathbb{C}(v)$. Let $W$ be the Weyl group and $w_{0}$ the element of $W$ of maximal length. Lusztig obtained a basis $B_{\mathbf{i}}$ of $U^{-}$of Poincaré-Birkhoff-Witt type for each reduced expression $\mathbf{i}$ of $w_{0}$ as a product of Coxeter generators of $W$. We shall call $B_{\mathbf{i}}$ a PBW-basis of $U^{-}$. The elements of $B_{\mathbf{i}}$ have form $F_{\mathbf{i}}^{\mathbf{c}}$ where c runs over the set of $N$-tuples of non-negative integers where $N=\ell\left(w_{0}\right)$.

Let $L$ be the $\mathbb{C}\left[v^{-1}\right]$-submodule of $U^{-}$spanned by the basis $B_{\mathbf{i}}$. This can be seen to be independent of the reduced expression $\mathbf{i}$ for $w_{0}$. Moreover the image of $B_{\mathbf{i}}$ under the projection

$$
L \longrightarrow L / v^{-1} L
$$

is also independent of the choice of $\mathbf{i}$. Now there is an involution of $U^{-}$which interchanges $v$ and $v^{-1}$. Let $\bar{L}$ be the image of $L$ under this involution. Then the map

$$
L \cap \bar{L} \longrightarrow L / v^{-1} L
$$

is bijective. The basis $B$ of $L \cap \bar{L}$ corresponding to the basis we have just described in $L / v^{-1} L$ is the canonical basis of $U^{-}$.

This canonical basis $B$ of $U^{-}$has many beautiful properties. For example let $L(\lambda)$ be the finite dimensional irreducible $U$-module with highest weight $\lambda$. Then $L(\lambda)$ has a highest weight vector $v_{\lambda}$ such that $L(\lambda)=U^{-} v_{\lambda}$. 
Lusztig showed that the set of elements $u v_{\lambda}$ for $u \in B$ which are non-zero form a basis for $L_{\lambda}$. Thus the canonical basis $B$ of $U^{-}$gives rise to bases of all finite dimensional irreducible highest weight modules $L(\lambda)$ simultaneously.

By specialising $v \rightarrow 1$ we obtain a canonical basis $\mathcal{B}$ of the enveloping algebra $\mathcal{U}^{-}$and bases for all finite dimensional irreducible $\mathcal{U}$-modules $\mathcal{L}(\lambda)$. Thus, although the existence of the canonical basis could only be proved in the context of quantum groups, this basis has striking applications to the classical representation theory of simple Lie algebras.

Lusztig described the geometrical significance of the canonical basis in terms of the intersection cohomology of certain algebraic varieties related to the theory of quivers. In describing this we shall assume for convenience that the Dynkin diagram is of simply-laced type. This Dynkin diagram $\Delta$ has a unique decomposition

$$
\Delta=\Delta^{\prime} \cup \Delta^{\prime \prime}
$$

into disjoint subsets, each of which corresponds to a set of mutually orthogonal simple roots. We may choose a reduced expression $\mathbf{i}$ of $w_{0}$ adapted to this decomposition of $\Delta$ and this gives rise to a PBW-basis $B_{\mathbf{i}}=\left\{F_{\mathbf{i}}^{\mathbf{c}}\right\}$ of $U^{-}$. Let $B=\left\{b_{\mathbf{c}}\right\}$ be the canonical basis of $U^{-}$. These two bases are related by

$$
b_{\mathbf{c}}=\sum_{\mathbf{c}^{\prime}} P_{\mathbf{c}^{\prime} \mathbf{c}} F_{\mathbf{c}^{\prime}}
$$

with $P_{\mathbf{c}^{\prime} \mathbf{c}} \in \mathbb{C}\left[v^{-1}\right]$.

Lusztig was able to describe a geometrical interpretation of the coefficients $P_{\mathbf{c}^{\prime} \mathbf{c}}$. To explain this we first make $\Delta$ into a quiver by introducing arrows from elements of $\Delta^{\prime}$ to elements of $\Delta^{\prime \prime}$. By Gabriel's theorem the indecomposable representations of this quiver $\Delta$ are in bijective correspondence with the set $\Phi^{+}$of positive roots. Thus any representation of $\Delta$ has form

$$
V=\bigoplus_{r_{i} \in \Phi^{+}} c_{i} V_{r_{i}}
$$

where $V_{\alpha}$ is the indecomposable representation corresponding to $\alpha \in \Phi^{+}$. Let $\left\{\alpha_{1}, \ldots, \alpha_{\ell}\right\}$ be the set of simple roots and $\left\{r_{1}, \ldots, r_{N}\right\}$ the set of positive roots. We have

$$
r_{i}=\sum_{j} m_{i j} \alpha_{j} \quad \text { with } \quad m_{i j} \geq 0
$$


The dimension vector of $V_{r_{i}}$ is

$$
\left(m_{i 1}, \ldots, m_{i \ell}\right)
$$

and the dimension vector of $V$ is $\mathbf{d}=\left(d_{1}, \ldots, d_{\ell}\right)$ where

$$
d_{j}=\sum_{i} c_{i} m_{i j}
$$

We consider the set of all representations $V$ of $\Delta$ with fixed dimension vector $\mathbf{d}$. These correspond to the orbits of a certain algebraic group $G(\mathbf{d})$ on an algebraic variety $E(\mathbf{d})$ where

$$
G(\mathbf{d})=\prod_{i} G L_{d_{i}}(\mathbb{C}), \quad E(\mathbf{d})=\bigoplus_{i \rightarrow j} \operatorname{Hom}\left(\mathbb{C}^{d_{i}}, \mathbb{C}^{d_{j}}\right) .
$$

The group $G(\mathbf{d})$ acts on the variety $E(\mathbf{d})$ with finitely many orbits, and these orbits $O_{\mathbf{c}}$ are in bijective correspondence with vectors $\mathbf{c}=\left(c_{i}\right)$ such that

$$
\sum_{i=1}^{N} c_{i} m_{i j}=d_{j}
$$

and so with representations $V$ of $\Delta$ with dimension vector $\mathbf{d}$.

The geometric interpretation of the coefficients $P_{\mathbf{c}^{\prime} \mathbf{c}}$ is as follows. If $P_{\mathbf{c}^{\prime} \mathbf{c}} \neq 0$ the orbits $O_{\mathbf{c}}, O_{\mathbf{c}^{\prime}}$ satisfy the condition

$$
O_{\mathbf{c}^{\prime}} \subset \bar{O}_{\mathbf{c}}
$$

and we have

$$
P_{\mathbf{c}^{\prime} \mathbf{c}}=\sum_{i \geq 0} \operatorname{dim} \mathbf{I H}_{O_{\mathbf{c}^{\prime}}}^{i}\left(\bar{O}_{\mathbf{c}}\right) v^{-i}
$$

Thus $P_{\mathbf{c}^{\prime} \mathbf{c}}$ is a polynomial in $v^{-1}$ whose coefficients are the dimensions of the local intersection cohomology groups of the variety $\bar{O}_{\mathbf{c}}$ at a point in $O_{\mathbf{c}^{\prime}}$.

\section{\$17. Total positivity in real reductive groups}

Lusztig used the canonical basis to generalize the theory of totally positive matrices to any connected reductive group which is split over $\mathbb{R}$. We recall that a matrix in $G L_{n}(\mathbb{R})$ is totally positive if all its minors are positive and totally non-negative if all its minors are non-negative. These conditions may be expressed in terms of the canonical basis as follows. 
Let $L(\lambda)$ be the irreducible finite dimensional $G L_{n}(\mathbb{R})$-module with highest weight $\lambda$ and $B(\lambda)$ be the canonical basis of $L(\lambda)$. Then an element $g \in G L_{n}(\mathbb{R})$ is totally positive if and only if, for each $\lambda$, the matrix representing $g$ with respect to the basis $B(\lambda)$ has all entries positive. Similarly $g$ is totally non-negative if and only if, for each $\lambda$, the entries of the matrix are all non-negative.

Now let $G$ be a connected reductive group of simply-laced type which is split over $\mathbb{R}$. We may use the above criteria involving the canonical basis to define a subset $G_{\geq 0}$ of totally non-negative elements and a subset $G_{>0}$ of totally positive elements of $G$. This enables the theory of totally positive and totally non-negative matrices to be generalized from $G L_{n}(\mathbb{R})$ to such group $G$ over $\mathbb{R}$.

Lusztig showed that $G_{\geq 0}$ is a closed subset of $G$ in the standard topology coming from $\mathbb{R}$ and that $G_{>0}$ is a dense open subset of $G_{\geq 0}$. All elements of $G_{>0}$ can be shown to be regular, semisimple and $\mathbb{R}$-split. Furthermore $G_{>0}$ is one of the connected components of the open subset

$$
B^{+} w_{0} B^{+} \cap B^{-} w_{0} B^{-}
$$

of $G$, where $B^{+}, B^{-}$are the Borel subgroups of $G$ corresponding to the positive and negative roots respectively.

Lusztig also gave definitions of totally positive and totally non-negative elements of the flag variety $\mathcal{B}$ of $G$. He showed that the two subsets

$$
\left\{u B^{+} u^{-1} ; u \in U_{>0}^{-}\right\} \quad\left\{u B^{-} u^{-1} ; u \in U_{>0}^{+}\right\}
$$

of $\mathcal{B}$ are equal, where $U^{+}, U^{-}$are the unipotent radicals of $B^{+}, B^{-}$and $U_{>0}^{+}, U_{>0}^{-}$are their sets of totally positive elements. This set is denoted by $\mathcal{B}_{>0}$ and $\mathcal{B}_{\geq 0}$ is defined as the closure of $\mathcal{B}_{>0}$ in $\mathcal{B}$. The subsets $\mathcal{B}_{>0}$ and $\mathcal{B}_{\geq 0}$ of the flag variety $\mathcal{B}$ can be shown to have some striking properties. For examples $\mathcal{B}_{\geq 0}$ has a decomposition into cells parametrised by pairs $w, w^{\prime}$ of elements of the Weyl group $W$ satisfying $w \leq w^{\prime}$. The cell corresponding to $w \leq w^{\prime}$ is diffeomorphic to $\mathbb{R}_{>0}^{\ell\left(w^{\prime}\right)-\ell(w)}$. The cell corresponding to the pair $1<w_{0}$ is $\mathcal{B}_{>0}$. In fact $\mathcal{B}_{>0}$ is one of the connected components of the variety of all Borel subgroups which are opposed both to $B^{+}$and to $B^{-}$.

Although we have assumed that $G$ is of simply-laced type this theory of total positivity was extended by Lusztig to groups of arbitrary type, by proving the simply-laced case first and then proceeding by descent to a non-simply laced group of fixed points under a suitable automorphism. 
Lusztig's 1993 book 'Introduction to Quantum Groups' is a highly original account of the structure and representation theory of quantized enveloping algebras, and of the subject of canonical bases and total positivity. Among other things his theory of based modules in the book readily shows that the tensor products of tilting modules remain titlting, a fundamental fact in the theory of tilting modules.

\section{$\S 18$. Modular representations of simple algebraic groups}

Let $G(K)$ be a simple simply-connected algebraic group over an algebraically closed field $K$ of prime characteristic $p$. Then the irreducible rational $G(K)$-modules are parametrised by dominant integral weights. For each such weight $\lambda \in X^{+}$let $L(\lambda)_{K}$ be the corresponding irreducible $G(K)$ module. In 1980 Lusztig formulated a conjecture for the character of $L(\lambda)_{K}$ when $p$ is sufficiently large.

In order to explain this conjecture we go back to the characteristic 0 situation. Let $G(\mathbb{C})$ be the corresponding type of algebraic group over $\mathbb{C}$ and $V(\lambda)$ the irreducible rational $G(\mathbb{C})$-module with highest weight $\lambda \in X^{+}$. Let $v_{\lambda}$ be a highest weight vector in $V(\lambda)$. Let $\mathfrak{g}=\operatorname{Lie} G$ and $\mathcal{U}$ be the universal enveloping algebra of $\mathfrak{g}$. There is a useful $\mathbb{Z}$-form $\mathcal{U}_{\mathbb{Z}}$ of $\mathcal{U}$ defined by Kostant. Let

$$
\begin{aligned}
V(\lambda)_{\mathbb{Z}} & =\mathcal{U}_{\mathbb{Z}} v_{\lambda} \\
V(\lambda)_{K} & =V(\lambda)_{\mathbb{Z}} \otimes K .
\end{aligned}
$$

Then $V(\lambda)_{K}$ is a $G(K)$-module, called a Weyl module. It has a unique maximal submodule, and its irreducible quotient is isomorphic to $L(\lambda)_{K}$. Lusztig's conjecture relates the characters of the $L(\lambda)_{K}$ to those of the $V(\lambda)_{K}$, which are given by Weyl's character formula.

The conjecture involves the action of the affine Weyl group on the lattice of weights. Let $X$ be the weight lattice and $X_{\mathbb{R}}$ the Euclidean space $X \otimes \mathbb{R}$. Let $\Phi$ be the set of roots of $G$. For each $\alpha \in \Phi, k \in \mathbb{Z}$ we define the affine hyperplane $H_{\alpha, k}$ by

$$
H_{\alpha, k}=\left\{\lambda \in X_{\mathbb{R}} ;(\lambda+\rho)\left(h_{\alpha}\right)=k p\right\}
$$

where $h_{\alpha}$ is the coroot of $\alpha$ and $\rho$ is the sum of the fundamental weights. Let $s_{\alpha, k}$ be the reflection in the affine hyperplane $H_{\alpha, k}$. The group $W_{a}$ of affine transformations of $X_{\mathbb{R}}$ generated by the $s_{\alpha, k}$ is isomorphic to the 
affine Weyl group. Its action on $X_{\mathbb{R}}$ is denoted by $w \cdot \lambda$. The connected components of $X_{\mathbb{R}}-\bigcup_{\alpha, k} H_{\alpha, k}$ are called alcoves. The set

$$
A_{1}=\left\{\lambda \in X_{\mathbb{R}} ; 0>(\lambda+\rho)\left(h_{\alpha}\right)>-p \text { for all } \alpha \in \Phi^{+}\right\}
$$

is an alcove, called the top antidominant alcove. If $A$ is an alcove so is $w . A$ for any $w \in W_{a}$. In fact each alcove has form $w \cdot A_{1}$ for a unique $w \in W_{a}$. We write

$$
A_{w}=w \cdot A_{1} .
$$

Thus the alcoves are labelled by the elements of the affine Weyl group.

We shall assume

$$
\rho\left(h_{\alpha_{0}}\right)<p
$$

where $h_{\alpha_{0}}$ is the highest coroot. This ensures that each alcove contains a weight in $X$. In particular $-2 \rho \in A_{1}$. We say that $w \in W_{a}$ is dominant if $w .(-2 \rho) \in X^{+}$. We say that $\lambda \in X$ is regular if $\lambda$ does not lie in any affine hyperplane $H_{\alpha, k}$. Suppose $\lambda \in X$ is dominant and regular. Let $w \in W_{a}$ be the element such that $\lambda \in A_{w}$. Then the Weyl module $V(\lambda)_{K}$ has composition factors $L(\mu)_{K}$ with multiplicities given by

$$
\left[V(\lambda)_{K}\right]=\sum_{\mu} d_{\mu \lambda}\left[L(\mu)_{K}\right]
$$

The only weights $\mu$ which can arise in the sum are those of the form $y w^{-1} \cdot \lambda$ where $y \in W_{a}$ is dominant. Thus

$$
\left[V(\lambda)_{K}\right]=\sum_{y \text { dominant }} d_{\mu \lambda}\left[L\left(y w^{-1} \cdot \lambda\right)_{K}\right] .
$$

By Jantzen's translation principal the decomposition numbers $d_{\mu \lambda}$ depend only upon $y$ and $w$. Thus

$$
\left[V(\lambda)_{K}\right]=\sum_{y \text { dominant }} d_{y, w}\left[L\left(y w^{-1} \cdot \lambda\right)_{K}\right] .
$$

for $\lambda \in A_{w}$. These equations may be inverted to give

$$
\left[L(\lambda)_{K}\right]=\sum_{y \text { dominant }} a_{y, w}\left[V\left(y w^{-1} \cdot \lambda\right)_{K}\right]
$$

for $\lambda \in A$, and certain $a_{y, w} \in \mathbb{Z}$. Suppose $w$ satisfies the so-called Jantzen condition

$$
-(w \rho)\left(h_{\alpha_{0}}\right) \leq p(p-h+2)
$$


which asserts, roughly speaking, that the alcove $A_{w}$ lies in the lowest dominant $p^{2}$-alcove of $X_{\mathbb{R}}$. ( $h$ is the Coxeter number of $G$ ). Then Lusztig's conjecture on modular representations asserts that

$$
a_{y, w}= \begin{cases}\varepsilon(y) \varepsilon(w) P_{y, w}(1) & \text { if } y \leq w \\ 0 & \text { otherwise }\end{cases}
$$

where $P_{y, w}(t)$ is a Kazhdan-Lusztig polynomial for the Coxeter group $W_{a}$ relative to the generator set $\left\{s_{\alpha}, s_{\alpha_{0},-1} \mid \alpha\right.$ simple $\}$.

This conjecture has some far-reaching consequences. In the first place Jautzen's translation principle asserts that if the above conjecture holds for $\lambda \in A_{w}$ it also holds for weights in the upper closure of $A_{w}$. Since each weight lies in the upper closure of some alcove, we obtain the characters of $L(\lambda)_{K}$ for singular weights $\lambda$ also. Secondly we obtain additional information from Steinberg's tensor product theorem. If $p$ is not too small the set $X_{1}$ of $p$-restricted weights given by

$$
X_{1}=\left\{\lambda \in X ; 0 \leq \lambda\left(h_{\alpha_{i}}\right) \leq p-1 \text { for } i=1, \ldots, \ell\right\}
$$

is included in the set of weights for which the conjecture is valid. If the characters of the $L(\lambda)_{K}$ are known for all $\lambda \in X_{1}$ they can be deduced for all $\lambda \in X^{+}$by Steinberg's tensor product theorem. Thus, under the given assumptions on $p$, Lusztig's conjecture would determine the characters of all irreducible $G(K)$-modules $L(\lambda)_{K}$.

Moreover Lusztig's conjecture would also determine the characters of the irreducible $K G^{F}$-modules, where $F$ is the Frobenius $q^{\text {th }}$-power map on $G(K)$ and $G^{F}=G(q)$ is the finite Chevalley group over the field of $q$ elements. There are $q^{\ell}$ irreducible $K G(q)$-modules where $\ell$ is the rank of $G$, and these modules are the $L(\lambda)_{K}$ for

$$
0 \leq \lambda\left(h_{\alpha_{i}}\right) \leq q-1 \text { for } i=1, \ldots, \ell .
$$

Progress towards a proof of Lusztig's conjecture on modular representations awaited the development of the theory of quantum groups. This conjecture is very similar to his conjecture about the characters of irreducible modules for quantum groups $U_{\zeta}$ at a $p^{\text {th }}$ root of unity, as described in Section 15. In fact Lusztig had proposed a programme in 1988 to relate three types of representation theory: the modular representations of simple algebraic groups, the representations of quantum groups at a $p^{\text {th }}$ root of unity, and the representations of certain affine Kac-Moody algebras. He 
proposed that the characters of the irreducible highest weight representations with the relevant negative central charge for the affine Kac-Moody algebras should be obtained by constructing an affine version of the proof of the original Kazhdan-Lusztig conjecture for Verma modules. This result should then lead to a knowledge of the irreducible representations of the quantum groups at a $p^{\text {th }}$ root of unity, and finally to a knowledge of the irreducible modular representations of simple algebraic groups.

Lusztig's programme led to a major development in the representation theory of algebraic groups, quantum groups, and affine Kac-Moody algebras in the last decade of the $20^{\text {th }}$ century. Thus the character formula for affine Kac-Moody algebras was proved by Kashiwara and Tanisaki, and the connection between the representation theory of affine Kac-Moody algebras and of quantum groups at a root of unity was proved by Kazhdan and Lusztig, as described in Section 15. The final step in Lusztig's programme involved a comparison of $U_{\zeta}$-modules and $G(K)$-modules by reduction modulo $p$. This was achieved in a lengthy paper by Andersen, Jantzen and Soergel in 1994, which has the consequence when combined with the other results of Lusztig's programme, that Lusztig's conjecture on modular representations is indeed valid if $p$ is sufficiently large. However it is not known how large $p$ must be for this to hold. Thus, apart from certain low rank cases, the characters of the irreducible modules $L(\lambda)_{K}$ remain unknown.

\section{§19. Modular representations of simple Lie algebras}

Now let $\mathfrak{g}$ be the Lie algebra of a simple algebraic group $G$ over an algebraically closed field of prime characteristic $p$. Consider irreducible representations of $\mathfrak{g}$. These are in bijective correspondence with irreducible representations of the enveloping algebra $\mathcal{U}(\mathfrak{g})$, and are all finite dimensional. $\mathfrak{g}$ has a $p$-operation $x \rightarrow x^{[p]}$ such that $x^{p}-x^{[p]}$ lies in the centre of $\mathcal{U}(\mathfrak{g})$ for each $x \in \mathfrak{g}$. Given any irreducible representation $\rho$ of $\mathcal{U}(\mathfrak{g})$ there is a linear map

$$
\chi: \mathfrak{g} \longrightarrow K
$$

called the $p$-character of $\rho$, such that

$$
\rho(x)^{p}-\rho\left(x^{[p]}\right)=\chi(x)^{p} I
$$

for all $x \in \mathfrak{g}$. Thus $\rho$ gives rise to an irreducible representation of the algebra $\mathcal{U}_{\chi}(\mathfrak{g})$ given by

$$
\mathcal{U}_{\chi}(\mathfrak{g})=\mathcal{U}(\mathfrak{g}) /\left\langle x^{p}-x^{[p]}-\chi(x)^{p} 1 ; x \in \mathfrak{g}\right\rangle
$$


$\mathcal{U}_{\chi}(\mathfrak{g})$ is called a reduced enveloping algebra. It is a finite dimensional algebra of dimension $p^{\text {dim } \mathfrak{g}}$. Thus each irreducible representation of $\mathfrak{g}$ determines an irreducible representation of $\mathcal{U}_{\chi}(\mathfrak{g})$ for a unique $p$-character $\chi$. The representation $\rho$ of $\mathfrak{g}$ is called restricted if $\chi=0$.

Now the characters of the irreducible restricted representations of $\mathfrak{g}$ can be derived from the irreducible characters of the algebraic group $G$ discussed in Section 18 in the case when Lusztig's conjecture is true. (For the $p$-restricted dominant highest weights there is in fact a bijective correspondence between them). Thus the discussion in Section 18 on modular representations of algebraic groups applies also to the restricted representations of $\mathfrak{g}$. However for non-restricted representations the situation is different.

In his survey article 'Representation theory in characteristic $p$ ' based on a lecture given in 1998, Lusztig formulated some conjectures on the irreducible representations of $\mathfrak{g}$ when $p$ is sufficiently large. Any such representation has a $p$-character $\chi$ which is a linear function on $\mathfrak{g}$, and which corresponds to an element $e \in \mathfrak{g}$ using the bijection between $\mathfrak{g}$ and its dual space given by the Killing form. It follows from the Jordan decomposition that it is sufficient to understand the case when $e$ is nilpotent. Moreover representations associated with nilpotent elements in the same orbit behave in the same way since $\mathcal{U}_{\chi}(\mathfrak{g})$ is isomorphic to $\mathcal{U}_{g . \chi}(\mathfrak{g})$ for each $g \in G$.

Now the set of irreducible $\mathfrak{g}$-modules with $p$-character corresponding to a given nilpotent element $e \in \mathfrak{g}$ admits an equivalence relation whose equivalence classes are called blocks. The generic blocks are those which contain the largest number of irreducible modules. Lusztig's formulation of the conjectures involves the connected reductive group $G_{\mathbb{C}}$ over $\mathbb{C}$ with the same root data as $G$. Let us denote the corresponding nilpotent element in the Lie algebra $\mathfrak{g}_{\mathbb{C}}$ of $G_{\mathbb{C}}$ by the same symbol $e$. He conjectured that the number of irreducible modules in such a generic block is the Euler characteristic of the algebraic variety $\mathcal{B}_{e}^{\mathbb{C}}$, the so-called Springer fibre, of all Borel subalgebras of $\mathfrak{g}_{\mathbb{C}}$ containing $e$. He also conjectured that the irreducible modules in such a generic block could be understood in the context of equivariant coherent sheaves on the variety $\mathcal{B}_{e}^{\mathbb{C}}$ (rather than perverse sheaves, which appeared in his earlier work). Let $(e, f, h)$ be the $\mathfrak{s l}_{2}$-triple in $\mathfrak{g}_{\mathbb{C}}, C$ a maximal torus of the simultaneous centralizer of $e, f$, $h$ in $G_{\mathbb{C}}$, and let $H$ be the torus given by

$$
H=C \times \mathbb{C}^{*} .
$$


There is a natural action of $H$ on $\mathcal{B}_{e}^{\mathbb{C}}$, which enable us to consider the group

$$
K_{H}\left(\mathcal{B}_{e}^{\mathbb{C}}\right)
$$

generated by the $H$-equivariant coherent sheaves on $\mathcal{B}_{e}^{\mathbb{C}}$ subject to the standard relations.

Now Lusztig showed how to define the concept of a canonical basis of $K_{H}\left(\mathcal{B}_{e}^{\mathbb{C}}\right)$, up to sign, as a $\mathbb{Z}\left[v, v^{-1}\right]$-module; proved the uniqueness of such a basis and conjectured its existence. He further conjectured that there is a natural bijection between irreducible modules in our generic block and elements in this canonical basis. Lusztig also made further conjectures on the irreducible modules, based on the following considerations.

In his series of papers on cells in affine Weyl groups, Lusztig had proved in 1989 the basic result, which he had conjectured earlier in 1980, that there is a natural bijection between the set of nilpotent orbits of a simple Lie algebra over $\mathbb{C}$ and the set of two-sided cells in the affine Weyl group of dual type. This bijection comes from the representation theory of extended affine Hecke algebras, for a parameter $q$ which is not a root of unity. As indicated in Sections 13 and 14 in our comments on Lusztig's version of the Deligne-Langlands conjecture, the irreducible representations of such an extended affine Hecke algebra are in bijective correspondence with triples $(s, e, \rho)$ coming from the algebraic group $G_{\mathbb{C}}^{*}$ of dual type, where

$s$ is a semisimple element of $G_{\mathbb{C}}^{*}$

$e$ is a nilpotent element of Lie $G_{\mathbb{C}}^{*}$

$\operatorname{Ad}(s) e=q e$

$\rho$ runs over a certain family of irreducible representations of $C(s, e) / C^{0}(s, e)$

The triples $(s, e, \rho)$ are taken up to conjugacy in $G_{\mathbb{C}}^{*}$. Now each irreducible representation of this extended affine Hecke algebra determines a two-sided cell in the affine Weyl group, by the Kazhdan-Lusztig theory. Moreover Lusztig was able to show that the two-sided cell given by the irreducible representation corresponding to $(s, e, \rho)$ is independent of $s$ and $\rho$ and depends only upon the orbit of the nilpotent element $e$. This gives rise to a bijection between two-sided cells in the affine Weyl group and nilpotent orbits in the Lie algebra of $G_{\mathbb{C}}^{*}$.

Returning now to consideration of the irreducible $\mathfrak{g}$-modules in a generic block, Lusztig further conjectures that there should be a surjective map from irreducible $\mathfrak{g}$-modules in the given generic block to left cells in the 
two-sided cell of the affine Weyl group corresponding to the nilpotent orbit containing $e$, such that two irreducible modules should map to the same left cell if and only if they are in the same orbit under the action of the component group of the centralizer of $e$.

Lusztig also made a conjecture which predicts the Cartan invariants of such a generic blocks, i.e., the multiplicities

$$
[P(L(i)): L(j)]
$$

of the irreducible modules $L(j)$ in their projective covers $P(L(i))$. This holds for $e=0$ if $p$ is sufficiently large by the solution of Lusztig's conjecture in Section 18. It also holds for $e$ regular nilpotent, and in many cases for $e$ subregular nilpotent due to Jantzen with an aid of Bezrukavnikov, Mirković and Rumynin.

Thus the form of a beautiful modular representation theory of simple Lie algebras is beginning to emerge from Lusztig's conjectures on this subjects. At the time of writing (April 2005), Lusztig's first conjecture on the number of irreducible modules in a generic block has been proved by Bezrukavnikov, Mirkovic and Rumynin, but the remaining conjectures relating to such modules are still unsettled, as far as the author is aware.

\section{§20. Summary}

It will be apparent from the above exposition that Lusztig's work is characterized by a very high degree of originality, an enormous breadth of subject matter, remarkable technical virtuosity, and great profundity in getting to the heart of the problems involved. It can be no exaggeration to say that George Lusztig is one of the great mathematicians of our time.

\section{Section 3}

\section{REFERENCES}

- G. Lusztig, The discrete series of $G L_{n}$ over a finite field, Ann. Math. Studies 81, Princeton Univ. Press, 1974, 99 pp.

- R. W. Carter and G. Lusztig, On the modular representations of the general linear and symmetric groups, Math. Zeit., 136 (1974), 193-242.

\section{Section 4}

- P. Deligne and G. Lusztig, Representations of reductive groups over finite fields, Ann. Math., 103 (1976), 103-161. 


\section{Section 5, 6, 7}

- R. B. Howlett and G. I. Lehrer, Induced cuspidal representations and generalized Hecke rings, Invent. Math., 58 (1980), 37-64.

- G. Lusztig, Characters of reductive groups over a finite field, Ann. Math. Studies 107, Princeton Univ. Press, 1984, 384 pp.

- R. W. Carter, Finite Groups of Lie Type (Conjugacy classes and complex characters), Wiley Classics Library, 1985, 544 pp.

- F. Digne and J. Michel, Representations of Finite Groups of Lie Type, London Math. Soc. Student Texts 21, 1991, 159 pp.

- M. Geck, Finite groups of Lie type, Representations of Reductive Groups (R. W. Carter and M. Geck, eds.), Cambridge University Press (1998), pp. 63-83.

\section{Section 8}

- D. Kazhdan and G. Lusztig, Representations of Coxeter groups and Hecke algebras, Inv. Math., 53 (1979), 165-184.

\section{Section 9}

- D. Kazhdan and G. Lusztig, Schubert varieties and Poincaré duality, Proc. Symp. Pure Math. 36, Amer. Math. Soc. (1980), pp. 185-203.

- G. Lusztig, Intersection cohomology methods in representation theory, Proc. Int. Congr. Math. Kyoto., Springer Verlag (1991), pp. 155-174.

\section{Section 10}

- A. Beilinson, J. Bernstein and P. Deligne, Faioceaux pervers, Analyse et topologie sur les éspaces singuliers (I), Astérisque 100, Paris, 1982.

- G. Lusztig, Intersection cohomology complexes on a reductive group, Inv. Math., 75 (1984), 205-272.

- G. Lusztig, Character sheaves I, Adv. in Math., 56 (1985), 193-237.

- G. Lusztig, Character sheaves II, Adv. in Math., 57 (1985), 226-265.

- G. Lusztig, Character sheaves III, Adv. in Math., 57 (1985), 266-315.

- G. Lusztig, Character sheaves IV, Adv. in Math., 59 (1986), 1-63.

- G. Lusztig, Character sheaves V, Adv. in Math., 61 (1986), 103-155.

- T. Shoji, On the Green polynomials of classical groups, Invent. Math., 74 (1983), 239-264.

- T. Shoji, Character sheaves and almost characters of reductive groups, Adv. in Math., 111 (1995), 244-313.

- T. Shoji, Character sheaves and almost characters of reductive groups II, Adv. in Math., 111 (1995), 314-354.

\section{Section 11}

- A. Beilinson and J. Bernstein, Localisation de g-modules, C. R. Acad. Sci. Paris, Sér I. Math., 292 (1981), 15-18. 
- J.-L. Brylinski and M. Kashiwara, Kazhdan-Lusztig conjecture and holonomic systems, Invent. Math., 64 (1981), 387-410.

\section{Section 12}

- G. Lusztig and D. Vogan, Singularities of closure of K-orbits on a flag manifold, Inv. Math., 71 (1983), 365-379.

\section{Section 13,14}

- G. Lusztig, Cuspidal local systems and graded Hecke algebras I, Publ. Math. IHES., 67 (1988), 145-202.

- G. Lusztig, Cuspidal local systems and graded Hecke algebras II, Representations of Groups. Canad. Math. Soc. Conf. Proc. 16, Amer. Math. Soc. (1995), pp. 217-275.

- G. Lusztig, Cuspidal local systems and graded Hecke algebras III, Representation Theory, 6 (2002), 202-242 (electronic).

- D. Kazhdan and G. Lusztig, Proof of the Deligne-Langlands conjecture for Hecke algebras, Inv. Math., 87 (1987), 153-215.

- G. Lusztig, Equivariant K-theory and representations of Hecke algebras, Proc. Amer. Math. Soc., 94 (1985), 337-342.

- G. Lusztig, Classification of unipotent representations of simple p-adic groups, Int. Math. Res. Notices, 1995, 517-589.

- G. Lusztig, Classification of unipotent representations of simple p-adic groups II, Representation Theory, 6 (2002), 243-289 (electronic).

\section{Section 15}

- G. Lusztig, Quantum deformations of certain simple modules over enveloping algebras, Adv. in Math., 70 (1988), 237-249.

- G. Lusztig, Quantum groups at roots of 1, Geom. Ded., 35 (1990), 89-114.

- D. Kazhdan and G. Lusztig, Affine Lie algebras and quantum groups, Int. Math. Res. Notices (1991), 21-29, In Duke Math. J., 62 (1991).

- M. Kashiwara and T. Tanisaki, Characters of the negative level highest weight modules for affine Lie algebras, Int. Math. Res. Notices, 3 (1994), 151-161.

\section{Section 16}

- G. Lusztig, Canonical bases arising from quantized enveloping algebras, J. Amer. Math. Soc., 3 (1990), 447-498.

- G. Lusztig, Canonical bases arising from quantized enveloping algebras II, Common trends in mathematics and quantum field theories, Prog. of Theor. Phys. Suppl. 102 (1990), pp. 175-201.

- M. Kashiwara, On crystal bases of the q-analogue of universal enveloping algebras, Duke Math. Jour., 63 (1991), 465-516.

- G. Lusztig, Introduction to quantized enveloping algebras, Prog. in Math. 105, Birkhäuser (1992), pp. 49-65. 


\section{Section 17}

- G. Lusztig, Total positivity in reductive groups, Lie theory and geometry: in honor of B. Kostant, Prog. in Math. 123, Birkhäuser (1994), pp. 531-568.

- G. Lusztig, Total positivity and canonical bases, Algebraic groups and Lie groups (G. Lehrer, ed.), Cambridge Univ. Press (1997), pp. 281-295.

- G. Lusztig, Introduction to quantum groups, Prog. in Math. 110, Bikhäuser, 1993, $341 \mathrm{pp}$.

\section{Section 18}

- G. Lusztig, Some problems in the representation theory of finite Chevalley groups, Proc. Symp. Pure Math. 37, Amer. Math. Soc. (1980), pp. 313-317.

- G. Lusztig, Modular representations and quantum groups, Cotemp. Math., 82 (1989), 59-77.

- H. H. Andersen, J. C. Jantzen and W. Soergel, Representations of quantum groups at a $p^{\text {th }}$ root of unity and of semisimple groups in characteristic $p$ : Independence of p, Astérisque 220, Paris, 1994.

\section{Section 19}

- G. Lusztig, Representation theory in characteristic p, Taniguchi Conf. on Math. Nara 1998, Adv. Stud. Pure Math. 31 (2001), pp. 167-178.

- G. Lusztig, Cells in affine Weyl groups I, Adv. Stud. Pure Math. 6 (1985), pp. 255-287.

- G. Lusztig, Cells in affine Weyl groups II, J. Algebra, 109 (1987), 536-548.

- G. Lusztig, Cells in affine Weyl groups III, J. Fac. Sci. Univ. Tokyo., 34 (1987), 223-243.

- G. Lusztig, Cells in affine Weyl groups IV, J. Fac. Sci. Univ. Tokyo., 36 (1989), 297-328.

- R. Bezrukavnikov, I. Mirkovic and D. Rumynin, Localization of modules for a semisimple Lie algebra in prime characteristic, to appear, preprint, math.RT/ 0205144.

Mathematics Research Centre

University of Warwick

Coventry CV4 7AL

United Kingdom

rwc@maths . warwick.ac.uk 